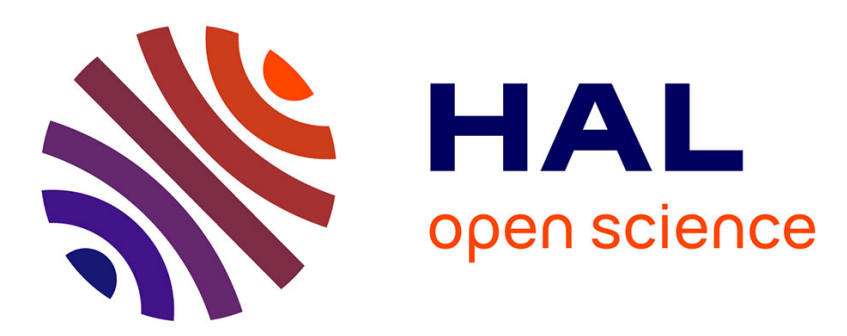

\title{
Enhancement of electromagnetic fields caused by interacting subwavelength cavities
}

Jean-François Babadjian, Eric Bonnetier, Faouzi Triki

\section{To cite this version:}

Jean-François Babadjian, Eric Bonnetier, Faouzi Triki. Enhancement of electromagnetic fields caused by interacting subwavelength cavities. Multiscale Modeling and Simulation: A SIAM Interdisciplinary Journal, 2010, 8 (4), pp.1383-1418. 10.1137/100787659 . hal-00463102

\section{HAL Id: hal-00463102 \\ https://hal.science/hal-00463102}

Submitted on 26 Mar 2010

HAL is a multi-disciplinary open access archive for the deposit and dissemination of scientific research documents, whether they are published or not. The documents may come from teaching and research institutions in France or abroad, or from public or private research centers.
L'archive ouverte pluridisciplinaire HAL, est destinée au dépôt et à la diffusion de documents scientifiques de niveau recherche, publiés ou non, émanant des établissements d'enseignement et de recherche français ou étrangers, des laboratoires publics ou privés. 


\title{
ENHANCEMENT OF ELECTROMAGNETIC FIELDS CAUSED BY INTERACTING SUBWAVELENGTH CAVITIES
}

\author{
JEAN-FRANÇOIS BABADJIAN, ERIC BONNETIER, AND FAOUZI TRIKI
}

\begin{abstract}
This article is devoted to the asymptotic analysis of the electromagnetic fields scattered by a perfectly conducting plane containing two sub-wavelength rectangular cavities. The problem is formulated through an integral equation, and a spectral analysis of the integral operator is performed. Using the generalized Rouche theorem on operator valued functions, it is possible to localize two types of resonances, symmetric and anti-symmetric, in a neighborhood of each zero of some explicit function, associated to the limiting geometry. For the symmetric modes, the fields in the cavities interact in phase, and the system of two cavities essentially acts as a dipole. In the anti-symmetric case, the fields oscillate in anti-phase, and the system behaves like a quadripole. Asymptotic expansions of the resonances, the far-field and the near-field are given.
\end{abstract}

KEYWORDS: Resonances, generalized Rouché theorem, integral operator, asymptotic expansion

2000 Mathematics Subject Classification: 31A10, 35J05, 47A56, 34E05, 78A45

\section{INTRODUCTION}

The interaction of light and rough metallic surfaces gives rise to fascinating phenomena, such as transmission of light through subwavelength apertures [9, 15, 13, 16], or such as Surface Enhancement Raman Scattering $[5,6,7,11]$. The optical excitation of resonant modes can lead to a concentration and localization of energy in volumes much smaller than $\lambda^{3}$, where $\lambda$ is the wavelength of the incident light. Potential applications are numerous, in particular to near field microscopy.

We are interested in metallic surfaces that contain parallel slits, which have been studied experimentally in $[5,6]$. Our objective is to understand the role played by the rugosity of the surfaces in the creation of resonant modes. The case of planar devices with rectangular cavities is particularly interesting. On the one hand, they can be manufactured with controled precision by current lithographic processes at the appropriate scales, and are widely used in opto-electronics. On the other hand, their simple geometry allows one to develop the mathematical analysis very far. Experimental results suggest that the amplification factors of the fields depend on the width of the cavities. In [8], the case of a half plane containing a single cavity of width $w$ was considered. The authors studied the asymptotic of the Green function as $w$ tends to zero using techniques based on integral representation $[3,18,10]$, which are well adapted to such geometry. The limiting Green function turns out to be that of an infinite half plane on which a dipole is placed. The present paper extends the analysis of [8] to two sub-wavelength cavities separated by a sub-wavelength distance, and studies the interaction between the cavities.

Let us briefly summarize our analysis and the main results. Due to geometrical considerations and to the choice of a time harmonic incident field $[5,8]$, the scattering problem can be reduced to a Helmholtz equation. Using the Green formula we reformulate the Helmholtz equation as a system of integral equations (2.11)-(2.12) defined on the aperture of the two cavities, for the normal derivatives of the solution $u$. The operator-valued matrix $\mathcal{S}_{w}(k)$ associated to the system depends on the width $w$ of the cavities and the frequency $k$ of the incident field. The kernels of the integral operators combine the Green functions of the Helmholtz operator in the rectangular cavities and in the upper half plane which are explicitly known. It is thus possible to derive a rigorous asymptotic expansion of $\mathcal{S}_{w}(k)$ with respect to $w$ (Lemma 3.2), and to ensure the invertibility of its dominant term in suitable fractional Sobolev spaces. Based on Fredholm theory we prove that the scattering resonances of the two cavities are exactly the poles of $\mathcal{S}_{w}^{-1}(k)$. Using the generalized Rouché Theorem for meromorphic operatorvalued functions (see [12] and the recent monograph [3]), and the asymptotic expansion of $\mathcal{S}_{w}^{-1}(k)$, we 
localize the resonances in the lower half complex plane (Theorem 4.10), and derive their asymptotic expansion (Theorem 4.11). Precisely, we prove the existence of two types of resonances. The first type corresponds to a symmetric mode with an imaginary part of order $O(w)$, the second type corresponds to an antisymmetric mode with a smaller imaginary part, of order $O\left(w^{3}\right)$, which leads to a much stronger electromagnetic enhancement (see Theorem 4.11). This partition of the resonances results from the near-field coupling of the cavities, and confirms the experimental results observed in [7]. Using again the generalized Rouché Theorem we derive the asymptotic expansion of the field $u$ far from and close to the resonances (Theorems 5.1-5.4). When the frequency is far from the resonances, the field is essentially the same as without cavities. However, when it is close to the resonances, the radiation pattern strongly depends on which mode is excited. Indeed, if the symmetric mode is active, then the scattered field $u$ behaves asymptotically like that of an infinite half plane on which a dipole is placed, as in the case of a single cavity. When the anti-symmetric mode is excited, the singularity is that of a quadripole. Finally, we perform an asymptotic expansion of the field inside the cavities (see Remark 5.7). In particular, we show that the field $u$ actually concentrates in the cavities when the frequency is close to the resonances. Moreover we prove that close to the symmetrical mode, the field has the same sign in both cavities, while close to the anti-symmetrical mode it has opposite signs.

The paper is organized as follows, we state the scattering problem in section 2, and reformulate it as an integral equation. Section 3 is devoted to the asymptotic expansion of $\mathcal{S}_{w}(k)$ as $w$ goes to zero. In section 4 , we derive useful qualitative properties of the operator-valued function $k \rightarrow \mathcal{S}_{w}(k)$, as well as the asymptotic expansion of $\mathcal{S}_{w}^{-1}(k)$ and the scattering resonances when $w$ is close to zero. Based on the previous results we give in section 5 , the asymptotic expansion of the scattered field in different regions of the scattering domain. Finally, in the appendix, we recall some results of Ghoberg and Sigal [12] on the operator version of the Residue theorem.

\section{Problem formulation}

Let $\Omega \subset \mathbb{R}^{2}$ be the domain defined by

$$
\Omega:=\Omega_{e} \cup C_{1} \cup C_{2} \cup \Gamma_{1} \cup \Gamma_{2},
$$

where $\Omega_{e}$ is the upper half-plane $\mathbb{R}_{+}^{2}, C_{i}, i=1,2$, are the rectangular cavities:

$$
C_{1}:=(-(d+1) w,-(d-1) w) \times(-h, 0), \quad C_{2}:=((d-1) w,(d+1) w) \times(-h, 0),
$$

and $\Gamma_{i}$ denotes the aperture of $C_{i}, i=1,2$, i.e.,

$$
\Gamma_{1}:=(-(d+1) w,-(d-1) w) \times\{0\}, \quad \Gamma_{2}:=((d-1) w,(d+1) w) \times\{0\} .
$$

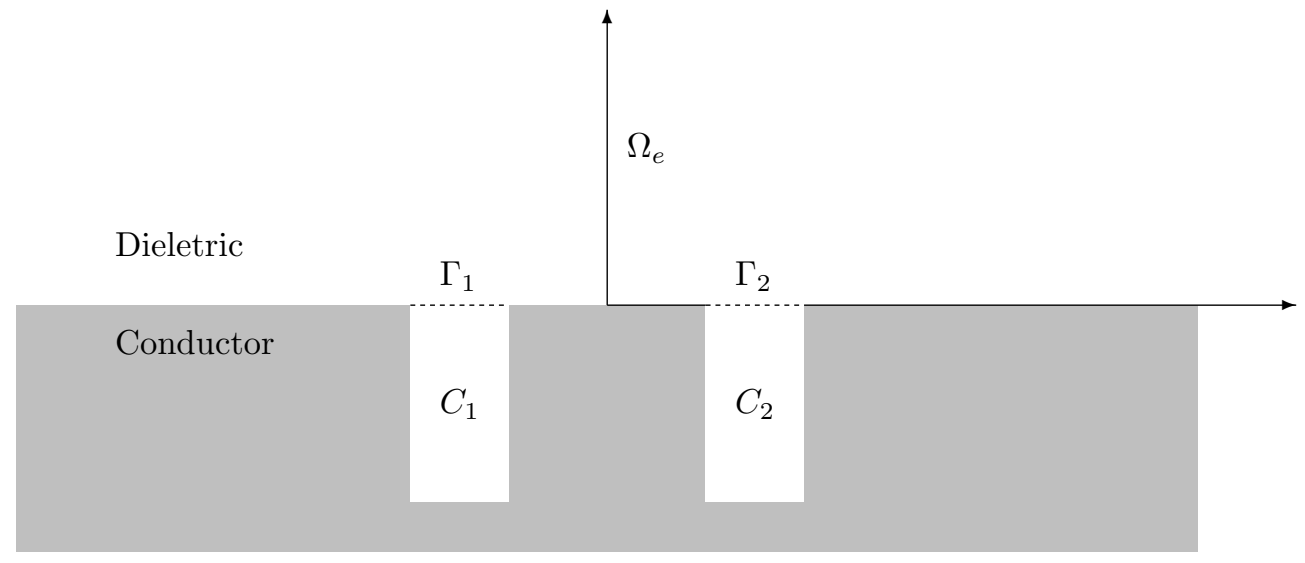

The cavities $C_{i}, i=1,2$ are illuminated by a source $f \in L^{2}\left(\Omega_{e}\right)$ with compact support in $\Omega_{e}$. In the harmonic regime, and under the same assumptions as in $[8,6]$, the Maxwell equations that govern 
the propagation of electromagnetic fields in the scattering domain $\Omega$ reduce to the following Helmholtz equation

$$
\left\{\begin{array}{l}
\Delta u+k^{2} u=f \quad \text { in } \Omega \\
\frac{\partial u}{\partial \nu}=0 \quad \text { on } \partial \Omega \\
\lim _{|x| \rightarrow+\infty} \sqrt{|x|}\left(\frac{\partial u}{\partial r}(x)-i k u(x)\right)=0
\end{array}\right.
$$

where $u(x)$ represents the component of the magnetic field in the transverse magnetic polarization. It is known that the problem $(2.1)$ has a unique solution whenever $\operatorname{Im}(k) \geq 0$ [2]. The mapping $R(k): f \rightarrow u$ defines an operator-valued function which is holomorphic in $\operatorname{Im}(k) \geq 0$. It has a meromorphic extension to the whole complex plane, except for a countable number of poles: These values of $k$ are the resonant frequencies. In other words, they are the values $k$ for which (2.1) has nontrivial solutions when $f=0$. The space of such non-trivial solutions, called characteristic functions, has finite dimension. When the pole $k_{j}$ is simple, the solution operator $R(k)$ can be factorized in the form

$$
R(k)=\frac{R_{-1, j}}{k-k_{j}}+R_{0, j}(k)
$$

where $R_{-1, j}$ is a finite rank operator, and where $R_{0, j}(k)$ is an operator-valued function which is holomorphic near $k_{j}$ [14]. The confinement of the electromagnetic fields around the cavities occurs at frequencies $k \in \mathbb{R}_{+}$close to $\operatorname{Re}\left(k_{j}\right)$, if the imaginary part $\operatorname{Im}\left(k_{j}\right)$ is small enough. In this case $\frac{\left\|R_{-1, j}\right\|}{\left|\operatorname{Im}\left(k_{j}\right)\right|}$ represents the factor of enhancement of the fields. Experimental results (see [5] and references therein) show that when the width of the cavities is smaller than the wavelength, the resonant frequencies are close to the real axis. In [8], the resonnant frequencies to a simple cavity have been studied, as the cavity width $w \rightarrow 0$. Precisely, it is proven there that $\operatorname{Im}\left(k_{j}\right)=O(w)$ as $w \rightarrow 0$. The imaginary part of the resonant frequencies also represents the lifetime of the confinement phenomena (see section 2.1 in [8]), which plays an important role in applications.

Recently, it was shown in [7] that a system of two deep identical cavities at subwavelength distance could produce resonant frequencies much closer to the real axis than those created by a simple cavity. Thus the optical excitation of such resonances can lead to a larger factor of concentration of the electromagnetic fields near the cavities. In this work, we analyse how the interplay between the fields in two cavities may cause such phenomena.

2.1. Fractional Sobolev spaces. Let $s \in \mathbb{R}$, we denote by $H^{s}(\mathbb{R})$ the space of tempered distributions $u \in \mathcal{S}^{\prime}(\mathbb{R})$ with Fourier transform $\widehat{u} \in L_{\text {loc }}^{2}(\mathbb{R})$, and

$$
\|u\|_{H^{s}(\mathbb{R})}^{2}:=\int_{\mathbb{R}}\left(1+|\xi|^{2}\right)^{s}|\widehat{u}(\xi)|^{2} d \xi<+\infty .
$$

Let $I$ be a bounded and open interval in $\mathbb{R}$. The sobolev space $H^{s}(I)$ is defined by

$$
H^{s}(I):=\left\{u \in\left(\mathcal{C}_{c}^{\infty}(I)\right)^{\prime}: u=\left.U\right|_{I} \text { for some } U \in H^{s}(\mathbb{R})\right\} .
$$

It is endowed with the norm

$$
\|u\|_{H^{s}(I)}=\inf \left\{\|U\|_{H^{s}(\mathbb{R})}: U \in H^{s}(\mathbb{R}),\left.\quad U\right|_{I}=u\right\} .
$$

It follows that $H^{s}(I)$ (resp. $H^{s}(\mathbb{R})$ ) equipped with the norm $\|\cdot\|_{H^{s}(I)}\left(\operatorname{resp} .\|\cdot\|_{H^{s}(\mathbb{R})}\right)$ is a Hilbert space. We also denote by $\widetilde{H}^{s}(I)$ the closure of $\mathcal{C}_{c}^{\infty}(I)$ in $H^{s}(\mathbb{R})$ so that $H^{-s}(I)=\left[\widetilde{H}^{s}(I)\right]^{\prime}$ and $\widetilde{H}^{-s}(I)=$ $\left[H^{s}(I)\right]^{\prime}$ (see [17, Theorem $\left.3.30(\mathrm{i})\right]$ ). Moreover, by [17, Theorem 3.29 (ii)] we have that

$$
\widetilde{H}^{s}(I)=\left\{u \in H^{s}(\mathbb{R}): \operatorname{Supp} u \subset \bar{I}\right\},
$$

and when $s \geq 0,[17$, Theorem 3.3] asserts that

$$
\widetilde{H}^{s}(I)=\left\{u \in L^{2}(I): \tilde{u} \in H^{s}(\mathbb{R})\right\},
$$

where $\tilde{u}$ denotes the extension of $u$ by zero outside $I$. 
In the sequel we will mostly be concerned with the cases $s= \pm 1 / 2$; in particular, it is proved (see [17, Theorem 3.30 (ii)]) that $H^{1 / 2}(I)$ can be identified to the space of functions $u \in L^{2}(I)$ satisfying

$$
\int_{I} \int_{I} \frac{|u(x)-u(y)|^{2}}{|x-y|^{2}} d x d y<+\infty
$$

If $\phi \in \widetilde{H}^{-1 / 2}(I)$ and $f \in H^{1 / 2}(I)$, we will denote by $\langle\phi, f\rangle$ the duality product between $\widetilde{H}^{-1 / 2}(I)$ and $H^{1 / 2}(I)$. Similarly for vector valued functions, the duality product between $\left[\widetilde{H}^{-1 / 2}(I)\right]^{2}$ and $\left[H^{1 / 2}(I)\right]^{2}$ will be denoted by $\langle\phi, f\rangle:=\left\langle\phi_{1}, f_{1}\right\rangle+\left\langle\phi_{2}, f_{2}\right\rangle$ whenever $\phi=\left(\phi_{1}, \phi_{2}\right) \in\left[\widetilde{H}^{-1 / 2}(I)\right]^{2}$ and $f=\left(f_{1}, f_{2}\right) \in$ $\left[H^{1 / 2}(I)\right]^{2}$.

2.2. The Green functions. Fix a source point $y \in \Omega_{e}$, and consider $G_{e}$ the Green function of the Helmholtz operator in $\Omega_{e}$ with a homogeneous Neumann boundary condition and a Sommerfeld radiation condition at infinity, i.e.,

$$
\left\{\begin{array}{l}
\Delta G_{e}(\cdot ; y)+k^{2} G_{e}(\cdot ; y)=\delta_{y}(.) \quad \text { in } \Omega_{e}, \\
\frac{\partial G_{e}}{\partial \nu}(\cdot ; y)=0 \quad \text { on } \partial \Omega_{e}, \\
\lim _{|x| \rightarrow+\infty} \sqrt{|x|}\left(\frac{\partial G_{e}}{\partial r}(x ; y)-i k G_{e}(x ; y)\right)=0 .
\end{array}\right.
$$

It is known that

$$
G_{e}(x ; y)=-\frac{i}{4} H_{0}^{(1)}(k|x-y|)-\frac{i}{4} H_{0}^{(1)}(k|x-\tilde{y}|),
$$

where $\tilde{y}=\left(y_{1}, y_{2}\right)$ is the symmetric of $y=\left(y_{1}, y_{2}\right)$ with respect to the $y_{1}$-axis, and $H_{0}^{(1)}$ stands for the Hankel function of first kind and zero order. We introduce the function $u_{e}(x) \in H_{\text {loc }}^{1}\left(\Omega_{e}\right)$ solution to

$$
\left\{\begin{array}{l}
\Delta u_{e}+k^{2} u_{e}=f \quad \text { in } \Omega_{e} \\
\frac{\partial u_{e}}{\partial \nu}=0 \quad \text { on } \partial \Omega_{e} \\
\lim _{|x| \rightarrow+\infty} \sqrt{|x|}\left(\frac{\partial u_{e}}{\partial r}(x)-i k u_{e}(x)\right)=0
\end{array}\right.
$$

which may be represented as

$$
u_{e}(x)=\int_{\Omega_{e}} G_{e}(x ; y) f(y) d y
$$

Let $G_{i}(i=1,2)$ be the Green function of the Helmholtz operator in the cavity $C_{i}$, with a homogeneous Neumann boundary condition, i.e.,

$$
\left\{\begin{array}{l}
\Delta G_{i}(\cdot ; y)+k^{2} G_{i}(\cdot ; y)=\delta_{y}(.) \quad \text { in } C_{i}, \\
\frac{\partial G_{i}}{\partial \nu}(\cdot ; y)=0 \quad \text { on } \partial C_{i} .
\end{array}\right.
$$

For $k^{2} \neq\left(\frac{m \pi}{2 w}\right)^{2}+\left(\frac{n \pi}{h}\right)^{2}$, the Green functions $G_{1}$ and $G_{2}$ have the following spectral decomposition

$$
G_{1}(x ; y)=\frac{2}{w h} \sum_{m, n=0}^{+\infty} \frac{\cos \left(\frac{m \pi}{2}\left(\frac{x_{1}}{w}+d+1\right)\right) \cos \left(\frac{m \pi}{2}\left(\frac{y_{1}}{w}+d+1\right)\right) \cos \left(n \pi\left(\frac{x_{2}}{h}+1\right)\right) \cos \left(n \pi\left(\frac{y_{2}}{h}+1\right)\right)}{k^{2}-\left(\frac{m \pi}{2 w}\right)^{2}-\left(\frac{n \pi}{h}\right)^{2}} .
$$

and

$$
G_{2}(x ; y)=\frac{2}{w h} \sum_{m, n=0}^{+\infty} \frac{\cos \left(\frac{m \pi}{2}\left(\frac{x_{1}}{w}-d+1\right)\right) \cos \left(\frac{m \pi}{2}\left(\frac{y_{1}}{w}-d+1\right)\right) \cos \left(n \pi\left(\frac{x_{2}}{h}+1\right)\right) \cos \left(n \pi\left(\frac{y_{2}}{h}+1\right)\right)}{k^{2}-\left(\frac{m \pi}{2 w}\right)^{2}-\left(\frac{n \pi}{h}\right)^{2}} .
$$

Remark 2.1. Since $u$ and $G_{i}, i=1,2$ depend on $w$ and $k$, we sometimes write $u(x ; w)$ and $G_{i}(x ; y ; w, k), i=$ 1,2 respectively in place of $u(x)$ and $G_{i}(x ; y), i=1,2$ to emphasize the dependence on these parameters. 
2.3. Integral representation. In this section, we derive an integral representation of $u(x)$ that is equivalent to the problem (2.1). Multiplying equation $(2.1)$ by $G_{e}(x ; y)$, integrating over $\Omega_{e}$ and using the Green formula in $\Omega_{e}$ implies that for every $y \in \Omega_{e}$

$$
\begin{aligned}
u_{e}(y)= & \int_{\Omega_{e}} f(x) G_{e}(x ; y) d x=\int_{\Omega_{e}}\left[\Delta u(x)+k^{2} u(x)\right] G_{e}(x ; y) d x \\
= & \int_{\Omega_{e}}\left[\Delta G_{e}(x ; y)+k^{2} G_{e}(x ; y)\right] u(x) d x \\
& +\int_{\partial \Omega_{e}}\left[\frac{\partial u}{\partial \nu}(x) G_{e}(x ; y)-\frac{\partial G_{e}}{\partial \nu}(x ; y) u(x)\right] d \sigma(x) \\
= & u(y)-\int_{\Gamma_{1} \cup \Gamma_{2}} \frac{\partial u}{\partial x_{2}}\left(x_{1}, 0\right) G_{e}\left(x_{1}, 0 ; y\right) d x_{1}
\end{aligned}
$$

where we used equations $(2.2)$ and $(2.1)$ in the last equality.

Similarly, multiplying $(2.1)$ by $G_{i}(x ; y)$, integrating over $C_{i}$ and applying the Green formula in $C_{i}$ together with equation (2.5), and noting that $f$ is supported in $\Omega_{e}$, leads to

$$
0=u(y)+\int_{\Gamma_{i}} \frac{\partial u}{\partial x_{2}}\left(x_{1}, 0\right) G_{i}\left(x_{1}, 0 ; y\right) d x_{1} \quad \text { for every } y \in C_{i} .
$$

Since $u \in H_{\mathrm{loc}}^{1}(\Omega)$ and $\Delta u=f-k^{2} u \in L_{\mathrm{loc}}^{2}(\Omega)$, it follows by elliptic regularity that $u \in H_{\text {loc }}^{2}(\Omega)$. Sobolev imbedding implies that $u$ is a continuous function on $\Gamma_{i}, i=1,2$. Hence, letting $y$ tend to $\Gamma_{1} \cup \Gamma_{2}$ in (2.7), letting $y$ tend to $\Gamma_{i}$ in (2.8), and taking the difference, we infer that for every $y_{1} \in \Gamma_{1}$

$$
\begin{aligned}
\int_{\Gamma_{1}} \frac{\partial u}{\partial x_{2}}\left(x_{1}, 0\right)\left[G_{1}+G_{e}\right]\left(x_{1}, 0 ; y_{1}, 0\right) d x_{1} \\
+\int_{\Gamma_{2}} \frac{\partial u}{\partial x_{2}}\left(x_{1}, 0\right) G_{e}\left(x_{1}, 0 ; y_{1}, 0\right) d x_{1}=-u_{e}\left(y_{1}, 0\right),
\end{aligned}
$$

and that for every $y_{1} \in \Gamma_{2}$

$$
\begin{aligned}
\int_{\Gamma_{2}} \frac{\partial u}{\partial x_{2}}\left(x_{1}, 0\right)\left[G_{2}+G_{e}\right]\left(x_{1}, 0 ;\right. & \left.y_{1}, 0\right) d x_{1} \\
& +\int_{\Gamma_{1}} \frac{\partial u}{\partial x_{2}}\left(x_{1}, 0\right) G_{e}\left(x_{1}, 0 ; y_{1}, 0\right) d x_{1}=-u_{e}\left(y_{1}, 0\right) .
\end{aligned}
$$

Next, we rescale both previous equations. To this end, we set

$$
\Gamma:=(-1,1),
$$

and for $x \in \Gamma$

$$
\left\{\begin{array} { r l } 
{ \phi _ { 1 } ( x ) } & { : = \frac { \partial u } { \partial x _ { 2 } } ( w x - w d , 0 ) , } \\
{ \phi _ { 2 } ( x ) } & { : = \frac { \partial u } { \partial x _ { 2 } } ( w x + w d , 0 ) , }
\end{array} \text { and } \left\{\begin{array}{rl}
g_{1}(x) & :=-\frac{1}{w} u_{e}(w x-w d, 0) \\
g_{2}(x) & :=-\frac{1}{w} u_{e}(w x+w d, 0) .
\end{array}\right.\right.
$$

From (2.9) and (2.10), we deduce that for every $y \in \Gamma$

$$
\int_{\Gamma}\left\{\left[G_{1}+G_{e}\right](w x-w d, 0 ; w y-w d, 0) \phi_{1}(x)+G_{e}(w x+w d, 0 ; w y-w d, 0) \phi_{2}(x)\right\} d x=g_{1}(y)
$$

and

$$
\int_{\Gamma}\left\{\left[G_{2}+G_{e}\right](w x+w d, 0 ; w y+w d, 0) \phi_{2}(x)+G_{e}(w x-w d, 0 ; w y+w d, 0) \phi_{1}(x)\right\} d x=g_{2}(y) .
$$

Let us define the $2 \times 2$ matrix-valued kernel

$$
s_{w}(x, y, k):=\left(\begin{array}{cc}
{\left[G_{1}+G_{e}\right](w x-w d, 0 ; w y-w d, 0)} & G_{e}(w x+w d, 0 ; w y-w d, 0) \\
G_{e}(w x-w d, 0 ; w y+w d, 0) & {\left[G_{2}+G_{e}\right](w x+w d, 0 ; w y+w d, 0)}
\end{array}\right) .
$$


Since $u \in H_{\text {loc }}^{1}(\Omega)$ and $\Delta u \in L_{\text {loc }}^{2}(\Omega)$, it follows that $\left.\frac{\partial u}{\partial x_{2}}\right|_{\Gamma_{i}} \in\left[H^{1 / 2}\left(\Gamma_{i}\right)\right]^{\prime}=\widetilde{H}^{-1 / 2}\left(\Gamma_{i}\right)$ and thus $\phi_{1}$ and $\phi_{2} \in \widetilde{H}^{-1 / 2}(\Gamma)$.

Consequently, it is natural to define the integral operator $\mathcal{S}_{w}(k):\left[\widetilde{H}^{-1 / 2}(\Gamma)\right]^{2} \rightarrow\left[H^{1 / 2}(\Gamma)\right]^{2}$ by

$$
\mathcal{S}_{w}(k) \phi(x):=\int_{\Gamma} s_{w}(x, y, k) \phi(y) d y, \quad \text { for every } \phi=\left(\phi_{1}, \phi_{2}\right) \in\left[\widetilde{H}^{-1 / 2}(\Gamma)\right]^{2} .
$$

\section{Asymptotic expansion}

In this section we derive an asymptotic expansion of the kernel $s_{w}$ and of the associated integral operator $\mathcal{S}_{w}(k)$. This can be achieved thanks to the explicit expression (2.13) of $s_{w}$ using standard tools of pseudodifferential analysis (see [20]). In the sequel, we fix $w_{0}>0$ and set $r_{0}:=\frac{\pi}{\left(2 w_{0}\right)}$. In the complex plane, $D_{r}(a):=\{z \in \mathbb{C}:|z-a|<r\}$ denotes the disc centered at $a$ and of of radius $r>0$. If $a=0$, we write $D_{r}:=D_{r}(0)$ and $D_{r}^{+}:=\left(\mathbb{C} \backslash \mathbb{R}_{-}\right) \cap D_{r}$.

Lemma 3.1. For every $(w, k) \in\left(0, w_{0}\right) \times D_{r_{0}}^{+}$the kernel $s_{w}$ has the following asymptotic expansion:

$$
s_{w}(x, y, k)=\theta_{w}(k)+s(x, y)+s_{1}(x, y) w+\sum_{n=2}^{+\infty} s_{n}(x, y, k) w^{n}+\sum_{n=2}^{+\infty} t_{n}(x, y, k) w^{n} \ln w,
$$

where

$$
\theta_{w}(k):=\left(\begin{array}{cc}
\frac{\alpha(k)}{w}+\delta_{2}+\frac{1}{\pi}(\ln k+\ln w) & \delta+\frac{1}{\pi}(\ln k+\ln w) \\
\delta+\frac{1}{\pi}(\ln k+\ln w) & \frac{\alpha(k)}{w}+\delta_{2}+\frac{1}{\pi}(\ln k+\ln w)
\end{array}\right),
$$

is a constant $2 \times 2$ matrix, $\alpha(k)$ is a complex function defined in (3.11),

$$
\begin{aligned}
s(x, y):= & \frac{1}{\pi} \ln \left[4|x-y|\left|\sin \left(\frac{\pi}{4}(x-y)\right)\right|\left|\sin \left(\frac{\pi}{4}(x+y+2)\right)\right|\right] \mathrm{I} \\
& +\left(\begin{array}{cc}
\delta_{1} & \frac{1}{\pi} \ln |x-y+2 d| \\
\frac{1}{\pi} \ln |x-y-2 d| & \delta_{1}
\end{array}\right) .
\end{aligned}
$$

is a matrix valued kernel independent of $k, \delta_{i}, i=1,2$ are constants to be fixed later (Remark(4.6)) satisfying $\delta_{1}+\delta_{2}=\delta, i=1,2$ where $\delta$ is the universal constant defined in (3.8).

$$
s_{1}(x, y):=-\frac{1}{h}\left(\frac{2}{3}-\frac{|x-y|+x+y+2}{4}+\frac{(x-y)^{2}+(x+y+2)^{2}}{8}\right) \mathrm{I} .
$$

Moreover, for every $n \geq 1$, there exists functions $f_{n}, g_{n}$ and $h_{n}$ defined in (3.7) and (3.15) such that

$$
\begin{aligned}
s_{2 n}(x, y, k):= & \left(f_{2 n}(x-y, k)+h_{2 n}(x-y, k)+h_{2 n}(x+y+2, k)\right) \mathrm{I} \\
& +\left(\begin{array}{cc}
0 & f_{2 n}(x-y+2 d, k) \\
f_{2 n}(x-y-2 d, k) & 0
\end{array}\right), \\
s_{2 n+1}(x, y, k) & :=\left(h_{2 n+1}(x-y, k)+h_{2 n+1}(x+y+2, k)\right) \mathrm{I},
\end{aligned}
$$

and

$$
t_{2 n}(x, y, k):=\left(\begin{array}{cc}
g_{2 n}(x-y, k) & g_{2 n}(x-y+2 d, k) \\
g_{2 n}(x-y-2 d, k) & g_{2 n}(x-y, k)
\end{array}\right), \quad t_{2 n+1}(x, y, k)=\left(\begin{array}{cc}
0 & 0 \\
0 & 0
\end{array}\right) .
$$

Finally, there exists a constant $C_{1}>0$ (that only depends on $w_{0}$ ) such that for every $k \in D_{r_{0}}^{+}$,

$$
\left\|s_{1} w+\sum_{n=2}^{+\infty} s_{n}(\cdot, \cdot, k) w^{n}+\sum_{n=2}^{+\infty} t_{n}(\cdot, \cdot, k) w^{n} \ln w\right\|_{\mathcal{C}^{0,1}(\bar{\Gamma} \times \bar{\Gamma})} \leq C_{1} w .
$$


Proof. We consider the asymptotic expansion of the Hankel function near zero (see [1, page 360]),

$$
H_{0}^{(1)}(z)=\left\{1+\frac{2 i}{\pi}\left[\ln \left(\frac{z}{2}\right)+\gamma\right]\right\}\left(\sum_{n=0}^{+\infty} \frac{\left(-z^{2} / 4\right)^{n}}{(n !)^{2}}\right)-\frac{2 i}{\pi} \sum_{n=1}^{+\infty}\left(\sum_{m=1}^{n} \frac{1}{m}\right) \frac{\left(-z^{2} / 4\right)^{n}}{(n !)^{2}}
$$

where $\gamma$ is the Euler constant. For every $t \in \mathbb{R}$, we thus obtain

$$
-\frac{i}{2} H_{0}^{(1)}(k w|t|)=\delta+\frac{1}{\pi}(\ln w+\ln k)+\frac{1}{\pi} \ln |t|+\sum_{n=1}^{+\infty} f_{2 n}(t, k) w^{2 n}+\sum_{n=1}^{+\infty} g_{2 n}(t, k) w^{2 n} \ln w
$$

where, for each $n \in \mathbb{N}^{*}$,

$$
\left\{\begin{aligned}
f_{2 n}(t, k) & :=\frac{(-1 / 4)^{n}}{\pi(n !)^{2}}\left(-\sum_{m=1}^{n} \frac{1}{m}+\pi \delta+\ln |t|+\ln k\right) k^{2 n} t^{2 n} \\
g_{2 n}(t, k) & :=\frac{(-1 / 4)^{n}}{\pi(n !)^{2}} k^{2 n} t^{2 n}
\end{aligned}\right.
$$

and where

$$
\delta:=-\frac{i}{2}+\frac{1}{\pi}(\gamma-\ln 2)
$$

Note that $g_{2 n}(\cdot, k) \in \mathcal{C}^{\infty}(\mathbb{R})$ for any $k \in \mathbb{C}$, while $g_{2 n}(t, \cdot)$ is analytic in $\mathbb{C}$. On the other hand, since the function $t \mapsto t^{2} \ln |t|$ is of class $\mathcal{C}^{1, \nu}(\mathbb{R})$ for any $\nu \in[0,1)$ (see [8]), it follows that $f_{2 n}(\cdot, k) \in$ $\mathcal{C}^{2 n-1, \nu}(\mathbb{R}) \cap \mathcal{C}^{\infty}(\mathbb{R} \backslash\{0\})$ and that $f_{2 n}(t, \cdot)$ is analytic in $\mathbb{C} \backslash \mathbb{R}$. . Moreover, there exists a constant $C>0$ (depending only on $w_{0}$ ) such that for every $(w, k) \in\left(0, w_{0}\right) \times D_{r_{0}}^{+}$,

$$
\sum_{n=1}^{+\infty}\left\|f_{2 n}(\cdot, k)\right\|_{\mathcal{C}^{0,1}([-4,4])} w^{2 n-2} \leq C \quad \text { and } \quad \sum_{n=1}^{+\infty}\left\|g_{2 n}(\cdot, k)\right\|_{\mathcal{C}^{0,1}([-4,4])} w^{2 n-2} \leq C .
$$

Consequently, the limits

$$
f(t, k, w):=\sum_{n=1}^{+\infty} f_{2 n}(t, k) w^{2 n} \quad \text { and } \quad g(t, k, w):=\sum_{n=1}^{+\infty} g_{2 n}(t, k) w^{2 n} \ln w
$$

exist and furthermore

$$
\|f(\cdot, k, w)\|_{\mathcal{C}^{0,1}([-4,4])} \leq C w^{2} \quad \text { and } \quad\|g(\cdot, k, w)\|_{\mathcal{C}^{0,1}([-4,4])} \leq C w^{2} \ln w .
$$

Note that provided $w \in\left(0, w_{0}\right)$, the first series in $(3.9)$ (as well as its first derivative with respect to $t$ ) converges uniformly as a function of $(t, k) \in[-4,4] \times D_{r_{0}}^{+}$. Consequently, $f(\cdot, k, w) \in \mathcal{C}^{1, \nu}([-4,4]) \cap$ $\mathcal{C}^{\infty}([-4,4] \backslash\{0\})$ for any $k \in D_{r_{0}}^{+}$, and the function $f(t, \cdot, w)$ is analytic in $D_{r_{0}}^{+}$for every $t \in[-4,4]$. Arguing similarly for the second series in $(3.9)$, we can show that $g(\cdot, k, w) \in \mathcal{C}^{\infty}([-4,4])$ for any $k \in D_{r_{0}}^{+}$, and that $g(t, \cdot, w)$ is analytic in $D_{r_{0}}^{+}$for every $t \in[-4,4]$. As consequence, we infer from the definition of $G_{e}$, that

$$
\begin{aligned}
G_{e}(w x-w d, 0 ; w y-w d, 0)= & G_{e}(w x+w d, 0 ; w y+w d, 0)=-\frac{i}{2} H_{0}^{(1)}(k w|x-y|) \\
= & \delta+\frac{1}{\pi}(\ln w+\ln k)+\frac{1}{\pi} \ln |x-y| \\
& +\sum_{n=1}^{+\infty} f_{2 n}(x-y, k) w^{2 n}+\sum_{n=1}^{+\infty} g_{2 n}(x-y, k) w^{2 n} \ln w
\end{aligned}
$$

and that

$$
\begin{aligned}
G_{e}(w x \pm w d, 0 ; w y \mp w d, 0)= & -\frac{i}{2} H_{0}^{(1)}(k w|x-y \pm 2 d|) \\
= & \delta+\frac{1}{\pi}(\ln w+\ln k)+\frac{1}{\pi} \ln |x-y \pm 2 d| \\
& +\sum_{n=1}^{+\infty} f_{2 n}(x-y \pm 2 d, k) w^{2 n}+\sum_{n=1}^{+\infty} g_{2 n}(x-y \pm 2 d, k) w^{2 n} \ln w .
\end{aligned}
$$


We now turn our attention to the Green functions inside the cavities. From the expression of $G_{1}$ and $G_{2}$, we have

$$
\begin{aligned}
G_{1}(w x-w d, 0 ; w y-w d, 0) & =G_{2}(w x+w d, 0 ; w y+w d, 0) \\
& =\frac{2}{w h} \sum_{m, n=0}^{+\infty} \frac{\cos \left(\frac{m \pi}{2}(x+1)\right) \cos \left(\frac{m \pi}{2}(y+1)\right)}{k^{2}-\left(\frac{m \pi}{2 w}\right)^{2}-\left(\frac{n \pi}{h}\right)^{2}} .
\end{aligned}
$$

We define

$$
\mathcal{R}_{m}(w, k):=\frac{2}{h} \sum_{n=0}^{+\infty} \frac{1}{k^{2}-\left(\frac{m \pi}{2 w}\right)^{2}-\left(\frac{n \pi}{h}\right)^{2}} .
$$

When $m=0$, we observe that $\mathcal{R}_{0}(w, k)=\alpha(k)$, where

$$
\alpha(k):=\frac{1}{k}\left(\frac{1}{k h}+\cot (k h)\right),
$$

while for $m \in \mathbb{N}^{*}, \mathcal{R}_{m}(w, k)$ has the following asymptotic expansion as $w / m$ tends to zero:

$$
\mathcal{R}_{m}(w, k)=-\frac{2}{\pi} \frac{w}{m}-\frac{4}{\pi^{2} h} \frac{w^{2}}{m^{2}}-\sum_{n=3}^{+\infty} p_{n}(k) \frac{w^{n}}{m^{n}},
$$

where $p_{n}(k)$ are suitable nonnegative constants which depend on $k$ in an analytic manner. Actually, one has

$$
\begin{aligned}
\mathcal{R}_{m}(w, k)= & -\frac{1}{k^{2} h} \sum_{n=1}^{+\infty}\left(\frac{2 w k}{m \pi}\right)^{2 n} \\
& -\frac{2 w}{m \pi}\left\{1+\sum_{n=1}^{+\infty} \frac{1}{n !}\left[\prod_{j=0}^{n-1}\left(\frac{1}{2}+j\right)\right]\left(\frac{2 w k}{m \pi}\right)^{2 n}\right\}\left\{\frac{2}{\left.1-e^{-2 h \sqrt{\left(\frac{m \pi}{2 w}\right)^{2}-k^{2}}}-1\right\},}\right.
\end{aligned}
$$

which indeed can be expanded as a series of $\frac{w}{m}$. Thus, from (3.12), there exists a constant $C^{\prime}>0$ (that only depends on $w_{0}$ ) such that

$$
\sum_{n=2}^{+\infty} p_{n}(k) \frac{w^{n}}{m^{n}} \leq C^{\prime} \sum_{n=2}^{+\infty}\left(\frac{2 w k}{m \pi}\right)^{n}
$$

Note that the previous series converges when $m \in \mathbb{N}^{*}, w<w_{0}$ and $k \in D_{r_{0}}$ (recall that $r_{0}=\pi /\left(2 w_{0}\right)$ ). Using (3.12) and the definition of $G_{1}$ and $G_{2}$, we immediately deduce that

$$
\begin{aligned}
G_{1}(w x-w d, 0 ; w y-w d, 0)= & G_{2}(w x+w d, 0 ; w y+w d, 0) \\
= & \frac{\alpha(k)}{w}-\frac{1}{\pi} \sum_{m=1}^{+\infty} \frac{\cos \left(\frac{m \pi}{2}(x-y)\right)+\cos \left(\frac{m \pi}{2}(x+y+2)\right)}{m} \\
& -\frac{2}{\pi^{2} h} w \sum_{m=1}^{+\infty} \frac{\cos \left(\frac{m \pi}{2}(x-y)\right)+\cos \left(\frac{m \pi}{2}(x+y+2)\right)}{m^{2}} \\
& -\sum_{n=2}^{+\infty} p_{n}(k) w^{n}\left(\sum_{m=1}^{+\infty} \frac{\cos \left(\frac{m \pi}{2}(x-y)\right)+\cos \left(\frac{m \pi}{2}(x+y+2)\right)}{m^{n+1}}\right) .
\end{aligned}
$$

As a consequence,

$$
\begin{aligned}
G_{1}(w x-w d, 0 ; w y-w d, 0)= & G_{2}(w x+w d, 0 ; w y+w d, 0) \\
= & \frac{\alpha(k)}{w}+\frac{1}{\pi} \ln \left[4\left|\sin \left(\frac{\pi}{4}(x-y)\right)\right|\left|\sin \left(\frac{\pi}{4}(x+y+2)\right)\right|\right] \\
& -\frac{w}{h}\left(\frac{2}{3}-\frac{|x-y|+x+y+2}{4}+\frac{(x-y)^{2}+(x+y+2)^{2}}{8}\right) \\
& +\sum_{n=2}^{+\infty}\left[h_{n}(x-y, k)+h_{n}(x+y+2, k)\right] w^{n},
\end{aligned}
$$


where we defined

$$
h_{n}(t, k):=-\sum_{m=1}^{+\infty} p_{n}(k) \frac{\cos \left(\frac{m \pi t}{2}\right)}{m^{n+1}} .
$$

From [8], we know that for each $k \in \mathbb{C}$, the function $h_{2}(\cdot, k) \in \mathcal{C}^{1, \nu}(\mathbb{R}) \cap \mathcal{C}^{\infty}(\mathbb{R} \backslash\{0\})$ for any $\nu \in[0,1)$, and thus by iteration, the functions $h_{n}(\cdot, k) \in \mathcal{C}^{n-1, \nu}(\mathbb{R}) \cap \mathcal{C}^{\infty}(\mathbb{R} \backslash\{0\})$ for every $n \geq 2$. Moreover the function $h_{n}(t, \cdot)$ is analytic on $\mathbb{C}$ for every $t \in \mathbb{R}$. Using (3.13), we observe that for $w<w_{0}$ and $k \in D_{r_{0}}$, then

$$
\begin{aligned}
\sum_{n=2}^{+\infty}\left\|h_{n}(\cdot, k)\right\|_{\mathcal{C}^{0,1}([-4,4])} w^{n-2} & \leq \frac{1}{w^{2}} \sum_{m=1}^{+\infty} \sum_{n=2}^{+\infty} p_{n}(k) \frac{w^{n}}{m^{n}} \leq \frac{C^{\prime}}{w^{2}} \sum_{m=1}^{+\infty} \sum_{n=2}^{+\infty}\left(\frac{2 w k}{m \pi}\right)^{n} \\
& \leq \frac{C^{\prime} \pi^{2}}{6 w^{2}} \sum_{n=2}^{+\infty}\left(\frac{2 w k}{\pi}\right)^{n} \leq C^{\prime \prime}
\end{aligned}
$$

for some constant $C^{\prime \prime}>0$ depending only on $w_{0}$. Hence we deduce that the limit

$$
h(t, k, w):=\sum_{n=2}^{+\infty} h_{n}(t, k) w^{n},
$$

exists and that

$$
\|h(\cdot, k, w)\|_{\mathcal{C}^{0,1}([-4,4])} \leq C^{\prime \prime} w^{2} .
$$

Since the series (3.16) is uniformly converging as a function of $(t, k) \in[-4,4] \times D_{r_{0}}$, it follows that $h(\cdot, k, w) \in \mathcal{C}^{1, \nu}([-4,4]) \cap \mathcal{C}^{\infty}([-4,4] \backslash\{0\})$ for every $k \in D_{r_{0}}$ and $h(t, \cdot, w)$ is analytic in $D_{r_{0}}$ for every $t \in[-4,4]$.

We define $\theta_{w}(k), s, s_{1}, s_{n}$ and $t_{n}$ (for $n \geq 2$ ) as in the statement of Lemma 3.1, and let

$$
\rho_{w}(x, y, k):=s_{1}(x, y) w+\sum_{n=2}^{+\infty} s_{n}(x, y, k) w^{n}+\sum_{n=2}^{+\infty} t_{n}(x, y, k) w^{n} \ln w .
$$

Thanks to (3.9) and (3.16), we deduce that for every $w \in\left(0, w_{0}\right)$, both previous series are uniformly converging in $\bar{\Gamma} \times \bar{\Gamma} \times D_{r_{0}}^{+}$as functions of $(x, y, k)$. Consequently $\rho_{w}(\cdot, \cdot, k) \in \mathcal{C}^{0,1}(\bar{\Gamma} \times \bar{\Gamma})$ for every $k \in D_{r_{0}}^{+}$and $\rho_{w}(x, y, \cdot)$ is analytic in $D_{r_{0}}^{+}$for every $(x, y) \in \bar{\Gamma} \times \bar{\Gamma}$. Finally, $s_{w}$ expands as announced in (3.1), and from (3.10) and (3.17) we immediately deduce (3.5).

For every $\phi \in\left[\mathcal{C}_{c}^{\infty}(\Gamma)\right]^{2}$, define the following integral operators

$$
\left\{\begin{aligned}
\Theta_{w}(k) \phi(x) & :=\theta_{w}(k) \int_{\Gamma} \phi(y) d y, \\
\mathcal{S} \phi(x) & :=\int_{\Gamma} s(x, y) \phi(y) d y, \\
\mathcal{S}_{1} \phi(x) & :=\int_{\Gamma} s_{1}(x, y) \phi(y) d y, \\
\mathcal{S}_{n}(k) \phi(x) & :=\int_{\Gamma} s_{n}(x, y, k) \phi(y) d y, \\
\mathcal{T}_{n}(k) \phi(x) & :=\int_{\Gamma} t_{n}(x, y, k) \phi(y) d y .
\end{aligned}\right.
$$

We now deduce from Lemma 3.1 an asymptotic expansion of the integral operator $\mathcal{S}_{w}(k)$.

Lemma 3.2. For every $(w, k) \in\left(0, w_{0}\right) \times D_{r_{0}}^{+}$, the operator $\mathcal{S}_{w}(k)$ admits the following asymptotic expansion:

$$
\mathcal{S}_{w}(k)=\boldsymbol{\Theta}_{w}(k)+\mathcal{S}+\mathcal{S}_{1} w+\sum_{n=2}^{+\infty} \mathcal{S}_{n}(k) w^{n}+\sum_{n=2}^{+\infty} \mathcal{T}_{n}(k) w^{n} \ln w,
$$


where $\mathcal{S}_{1}, \mathcal{S}_{n}(k)$ and $\mathcal{T}_{n}(k)$ are compact from $\left[\widetilde{H}^{-1 / 2}(\Gamma)\right]^{2}$ to $\left[H^{1 / 2}(\Gamma)\right]^{2}$ and

$$
\left\|\mathcal{S}_{1} w+\sum_{n=2}^{+\infty} \mathcal{S}_{n}(k) w^{n}+\sum_{n=2}^{+\infty} \mathcal{T}_{n}(k) w^{n} \ln w\right\| \leq C_{2} w,
$$

for some constant $C_{2}>0$ depending only on $w_{0}$.

Proof. Let $\chi \in \mathcal{C}_{c}^{\infty}(\mathbb{R} ;[0,1])$ be a cut-off function satisfying $\chi(t)=1$ for every $t \in \Gamma$. For any integer $n \geq 2$ and every $(x, y) \in \mathbb{R} \times \mathbb{R}$, define

$$
\left\{\begin{array}{l}
\tilde{s}_{n}(x, y, k):=\chi(x) \chi(y) s_{n}(x, y, k), \\
\tilde{t}_{n}(x, y, k):=\chi(x) \chi(y) t_{n}(x, y, k) .
\end{array}\right.
$$

Denoting by $\sigma_{n}$ and $\tau_{n}$ the symbols of $\tilde{s}_{n}$ and $\tilde{t}_{n}$ respectively, on can check that $\sigma_{n}$ and $\tau_{n}$ belong to the class of symbols $S_{1,0}^{-n}$ and thus (see [20, Chapter II]), it follows that the associated integral operators $\tilde{\mathcal{S}}_{n}(k)$ and $\tilde{\mathcal{T}}_{n}(k)$ defined, for every $\phi \in\left[\mathcal{C}_{c}^{\infty}(\mathbb{R})\right]^{2}$, by

$$
\tilde{\mathcal{S}}_{n}(k) \phi(x):=\int_{\mathbb{R}} \tilde{s}_{n}(x, y, k) \phi(y) d y \quad \text { and } \quad \tilde{\mathcal{T}}_{n}(k) \phi(x):=\int_{\mathbb{R}} \tilde{t}_{n}(x, y, k) \phi(y) d y
$$

are bounded from $\left[H^{-1 / 2}(\mathbb{R})\right]^{2}$ to $\left[H^{n-1 / 2}(\mathbb{R})\right]^{2}$. As a consequence, the operators $\mathcal{S}_{n}(k)$ and $\mathcal{T}_{n}(k)$ defined above are bounded from $\left[\widetilde{H}^{-1 / 2}(\Gamma)\right]^{2}$ to $\left[H^{n-1 / 2}(\Gamma)\right]^{2}$, and using the compact imbedding of $H^{n-1 / 2}(\Gamma)$ into $H^{1 / 2}(\Gamma)$ for $n \geq 2$, it follows that they are actually compact from $\left[\widetilde{H}^{-1 / 2}(\Gamma)\right]^{2}$ to $\left[H^{1 / 2}(\Gamma)\right]^{2}$. From [8] we also know that the integral operator $\mathcal{S}_{1}$ is compact from $\left[\widetilde{H}^{-1 / 2}(\Gamma)\right]^{2}$ to $\left[H^{1 / 2}(\Gamma)\right]^{2}$. Moreover, thanks to (3.1) and the Dominated Convergence Theorem, the operator $\mathcal{S}_{w}(k)$ has the expected asymptotic expansion for $(w, k) \in\left(0, w_{0}\right) \times D_{r_{0}}^{+}$. Finally, according to (3.5), the operator

$$
\mathcal{R}_{w}(k):=\mathcal{S}_{1} w+\sum_{n=2}^{+\infty} \mathcal{S}_{n}(k) w^{n}+\sum_{n=2}^{+\infty} \mathcal{T}_{n}(k) w^{n} \ln w,
$$

is an integral operator with kernel $\rho_{w}(x, y, k)$ given by $(3.18)$, and we have $\left\|\mathcal{R}_{w}(k)\right\| \leq C_{2} w$ where $C_{2}>0$ is a constant depending on $w_{0}$ and $C_{1}$.

\section{Asymptotic of $\mathcal{S}_{w}^{-1}(k)$ And of the REsonanCES}

Let $\widetilde{S}:\left[\widetilde{H}^{-1 / 2}(\Gamma)\right]^{2} \rightarrow\left[H^{1 / 2}(\Gamma)\right]^{2}$ be the integral operator associated to the kernel

$$
\frac{1}{\pi} \ln \left[|x-y|\left|\sin \left(\frac{\pi}{4}(x-y)\right)\right|\left|\sin \left(\frac{\pi}{4}(x+y+2)\right)\right|\right] \mathrm{I} .
$$

The following result is proved in [8](see Lemma 5.1).

Lemma 4.1. The operator $\widetilde{S}$ is invertible from $\left[\widetilde{H}^{-1 / 2}(\Gamma)\right]^{2}$ to $\left[H^{1 / 2}(\Gamma)\right]^{2}$.

Theorem 3.2 implies that for every $(w, k) \in\left(0, w_{0}\right) \times D_{r_{0}}^{+}$, we have $\mathcal{S}_{w}(k)=\boldsymbol{\Theta}_{w}(k)+\mathcal{S}+\mathcal{R}_{w}(k)$ where

$$
\boldsymbol{\Theta}_{w}(k)=\theta_{w}(k)\left(\begin{array}{c}
\left\langle\cdot, e_{1}\right\rangle \\
\left\langle\cdot, e_{2}\right\rangle
\end{array}\right),
$$

the matrix $\theta_{w}(k)$ is defined in $(3.2)$, and $e_{1}=(1,0), e_{2}=(0,1)$.

Theorem 4.2. The operator valued function $k \mapsto \mathcal{S}_{w}(k)$ is finitely meromorphic and of Fredholm type in $\mathbb{C} \backslash \mathbb{R}_{-}$, its poles are $\left\{ \pm\left(\left(\frac{n \pi}{h}\right)^{2}+\left(\frac{m \pi}{2 w}\right)^{2}\right)^{\frac{1}{2}}: n, m \in \mathbb{N}\right\}$. The operator $\mathcal{S}_{w}(k)$ is invertible from $\left[\widetilde{H}^{-1 / 2}(\Gamma)\right]^{2}$ to $\left[H^{1 / 2}(\Gamma)\right]^{2}$ for $\operatorname{Im}(k) \geq 0$. Moreover the operator valued function $k \mapsto \mathcal{S}_{w}^{-1}(k)$ is finitely meromorphic in $\mathbb{C} \backslash \mathbb{R}_{-}$and its poles are exactly the resonances of the open cavities.

Proof. By the expression (2.13) of the kernel $s_{w}$, it is clear that the poles of $\mathcal{S}_{w}(k)$ are exactly $\left\{ \pm\left(\left(\frac{n \pi}{h}\right)^{2}+\right.\right.$ $\left.\left.\left(\frac{m \pi}{2 w}\right)^{2}\right)^{\frac{1}{2}}: n, m \in \mathbb{N}\right\}$. Expanding the operator $\mathcal{S}_{w}(k)$ as a Laurent series around each of these poles 
implies that the range of the operator that consists in the multiplication by $\frac{1}{k \pm\left(\left(\frac{n \pi}{h}\right)^{2}+\left(\frac{m \pi}{2 w}\right)^{2}\right)^{\frac{1}{2}}}$ is of dimension two. The range of that operator is actually the vector space spanned by the function

$$
\cos \left(\frac{m \pi}{2}(x+1)\right)\left(e_{1}+e_{2}\right), \quad x \in \Gamma .
$$

This implies that $\mathcal{S}_{w}(k)$ is finitely meromorphic in $\mathbb{C} \backslash \mathbb{R}_{-}$.

Now, according to Lemma 3.2, the only term independent of $k$ in the previous Laurent series, is the operator

We remark that the following kernel

$$
\mathcal{A}_{0}:=\left(\begin{array}{cc}
\delta_{2}+\frac{\ln w}{\pi} & \delta+\frac{\ln w}{\pi} \\
\delta+\frac{\ln w}{\pi} & \delta_{2}+\frac{\ln w}{\pi}
\end{array}\right)\left(\begin{array}{c}
\left\langle., e_{1}\right\rangle \\
\left\langle., e_{2}\right\rangle
\end{array}\right)+\mathcal{S}+\mathcal{S}_{1} w
$$

$$
\left(\begin{array}{cc}
\delta_{1}+\ln (4) & \frac{1}{\pi} \ln |x-y+2 d| \\
\frac{1}{\pi} \ln |x-y-2 d| & \delta_{1}+\ln (4)
\end{array}\right)
$$

belongs to $C^{\infty}(\bar{\Gamma} \times \bar{\Gamma})$ and so the corresponding integral operator is compact from $\left[\widetilde{H}^{-1 / 2}(\Gamma)\right]^{2}$ to $\left[H^{1 / 2}(\Gamma)\right]^{2}$. It follows that $\mathcal{S}$ is a compact perturbation of $\tilde{S}$ which is invertible from $\left[\widetilde{H}^{-1 / 2}(\Gamma)\right]^{2}$ to $\left[H^{1 / 2}(\Gamma)\right]^{2}$ (Lemma 4.1). Since $\mathcal{S}_{1}$ is compact (Lemma 3.2) the operator $\mathcal{A}_{0}$ is also a compact perturbation of $\tilde{S}$ and so it is a Fredholm operator of index zero. Consequently, the operator valued function $k \mapsto \mathcal{S}_{w}(k)$ is of Fredholm type of index zero as well (see the appendix). The invertibility of the operator $\mathcal{S}_{w}(k)$ for $\operatorname{Im}(k) \geq 0$ is a consequence of the fact that the equation (2.1) admits a unique solution for such frequencies $k$. Finally, we deduce from the Steinberg Theorem (Theorem 6.3) that the operator valued function $k \mapsto \mathcal{S}_{w}^{-1}(k)$ is finitely meromorphic in $\mathbb{C} \backslash \mathbb{R}_{-}$and its poles are exactly the resonances of the open cavities.

Let $\widehat{S}:\left[\widetilde{H}^{-1 / 2}(\Gamma)\right]^{2} \rightarrow\left[H^{1 / 2}(\Gamma)\right]^{2}$ be the integral operator with kernel

$$
\frac{1}{\pi} \ln \left[4|x-y|\left|\sin \left(\frac{\pi}{4}(x-y)\right)\right|\left|\sin \left(\frac{\pi}{4}(x+y+2)\right)\right|\right] \mathrm{I}+\left(\begin{array}{cc}
0 & \frac{1}{\pi} \ln |x-y+2 d| \\
\frac{1}{\pi} \ln |x-y-2 d| & 0
\end{array}\right) .
$$

Let $\widetilde{H}_{0}^{-1 / 2}(\Gamma)$ denotes the space of functions $\varphi$ in $\widetilde{H}^{-1 / 2}(\Gamma)$ satisfying $\int_{\Gamma} \varphi(x) d x=0$.

Lemma 4.3. $\widehat{S}$ is Fredholm of index zero on $\left[\widetilde{H}^{-1 / 2}(\Gamma)\right]^{2}$. In addition, $\widehat{S}$ is coercive on $\left[\widetilde{H}_{0}^{-1 / 2}(\Gamma)\right]^{2}$, i.e.,

$$
-\langle\varphi, \widehat{S} \varphi\rangle \geq C\|\varphi\|_{\left[\widetilde{H}^{-1 / 2}(\Gamma)\right]^{2}}^{2}, \quad \forall \varphi \in\left[\widetilde{H}_{0}^{-1 / 2}(\Gamma)\right]^{2},
$$

where $C>0$ is a fixed constant.

Proof. The operator $\widehat{S}$ is a compact perturbation of $\widetilde{S}$ and so is Fredholm of index zero on $\left[\widetilde{H}^{-1 / 2}(\Gamma)\right]^{2}$. Let $\varphi=\left(\varphi_{1}, \varphi_{2}\right) \in\left[\widetilde{H}^{-1 / 2}(\Gamma)\right]^{2}$ and define

$$
\begin{aligned}
\varphi_{1, w}(x) & :=\frac{1}{w} \varphi_{1}\left(\frac{x}{w}+d\right) & & x \in \Gamma_{1}, \\
\varphi_{2, w}(x) & :=\frac{1}{w} \varphi_{2}\left(\frac{x}{w}-d\right) & x & \in \Gamma_{2} .
\end{aligned}
$$

We consider the solution $v$ to the following Helmholtz equation

$$
\left\{\begin{array}{l}
\Delta v+k^{2} v=0 \quad \text { in } \Omega_{e} \cup C_{1} \cup C_{2}, \\
\left.\partial_{x_{2}} v\right|_{+}=\left.\partial_{x_{2}} v\right|_{-}=\varphi_{1, w} \text { on } \Gamma_{1} \\
\left.\partial_{x_{2}} v\right|_{+}=\left.\partial_{x_{2}} v\right|_{-}=\varphi_{2, w} \text { on } \Gamma_{2}, \\
\frac{\partial v}{\partial \nu}=0 \quad \text { on } \partial \Omega, \\
\lim _{|x| \rightarrow+\infty} \sqrt{|x|}\left(\frac{\partial v}{\partial r}(x)-i k v(x)\right)=0 .
\end{array}\right.
$$


Multiplying $v$ in the equation (4.2) by $G_{e}(x, y)$ and integrating by parts we obtain

$$
v(x)=\sum_{i=1}^{2} \int_{\Gamma_{i}} G_{e}(x, y) \varphi_{i, w}(y) d \sigma(y), \quad x \in \Omega_{e} .
$$

The Green formula inside the cavities yields

$$
v(x)=-\int_{\Gamma_{i}} G_{i}(x, y) \varphi_{i, w}(y) d \sigma(y), \quad x \in C_{i}, \quad i=1,2 .
$$

Taking the difference between the traces of the equations (4.3) and (4.4) on both sides of $\Gamma_{i}$, we obtain

$$
[v(x)]=\sum_{i=1}^{2} \int_{\Gamma_{i}}\left(G_{e}(x, y)+\delta_{i j} G_{i}(x, y)\right) \varphi_{i, w}(y) d \sigma(y), \quad x \in \Gamma_{j}, j=1,2,
$$

where $[v(x)]=\left.v\right|_{+}-\left.v\right|_{-}$and $\delta_{i j}$ is th Kronecker's delta. Now, we fix $k=i=\sqrt{-1}$. Due to the explicit expression of $G_{e}$ (equation (2.3)) one can easily check that when $k=i$, the function $v(x)$ belongs to $H^{1}\left(\Omega_{e}\right)$. Thus, multiplying the equation (4.2) by $\bar{v}$ and integrating over $\Omega_{e}$ leads to

$$
\int_{\Omega_{e}}|\nabla v(x)|^{2} d x+\int_{\Omega_{e}}|v(x)|^{2} d x=-\left.\sum_{i=1}^{2} \int_{\Gamma_{i}} \varphi_{i, w}(x) \bar{v}\right|_{+}(x) d \sigma(x) .
$$

Similarly, multiplying the equation (4.2) by $\bar{v}$ and integrating over $C_{i}$ implies

$$
\int_{C_{i}}|\nabla v(x)|^{2} d x+\int_{C_{i}}|v(x)|^{2} d x=\left.\int_{\Gamma_{i}} \varphi_{i, w}(x) \bar{v}\right|_{-}(x) d \sigma(x) .
$$

Adding the equations (4.6) and (4.7) we obtain

$$
\int_{\Omega}|\nabla v(x)|^{2} d x+\int_{\Omega}|v(x)|^{2} d x=-\sum_{i=1}^{2} \int_{\Gamma_{i}} \varphi_{i, w}(x) \overline{[v(x)]} d \sigma(x) .
$$

Replacing $[v(x)]$ by its expression in (4.5) and rescaling the right hand term in the last equation with respect to $w$, we find

$$
\int_{\Omega}|\nabla v(x)|^{2} d x+\int_{\Omega}|v(x)|^{2} d x=-\left\langle\varphi, \mathcal{S}_{w}(i) \varphi\right\rangle .
$$

On the other hand we have (see [17])

$$
C\|\varphi\|_{\left[\widetilde{H}^{-1 / 2}(\Gamma)\right]^{2}}^{2}=C\left\|\partial_{x_{2}} v\right\|_{H^{-1 / 2}\left(\partial \Omega_{e}\right)}^{2} \leq \int_{\Omega_{e}}|\nabla v(x)|^{2} d x+\int_{\Omega_{e}}|v(x)|^{2} d x,
$$

where $C>0$ is a constant independent of $w$. Combining the last two equalities yield

$$
-\left\langle\varphi, \mathcal{S}_{w}(i) \varphi\right\rangle \geq C\|\varphi\|_{\left[\widetilde{H}^{-1 / 2}(\Gamma)\right]^{2}}^{2} .
$$

Since $\varphi \in\left[\widetilde{H}_{0}^{-1 / 2}(\Gamma)\right]^{2}$, Lemma 3.2 implies

$$
S_{w}(i) \varphi \rightarrow \widehat{S} \varphi, \quad \text { as } \quad w \rightarrow 0 .
$$

Taking the limit of the equation (4.9) as $w$ tends to zero we obtain the desired result.

Lemma 4.4. Let $T:\left[\widetilde{H}^{-1 / 2}(\Gamma)\right]^{2} \times \mathbb{R}^{2} \rightarrow\left[H^{1 / 2}(\Gamma)\right]^{2} \times \mathbb{R}^{2}$ be the integral operator defined by

$$
T(\psi, a):=\left(\widehat{S} \psi-a, \int_{\Gamma} \psi(x) d x\right) .
$$

Then, $T$ is invertible.

Proof. Since $\widehat{S}$ is Fredholm of index zero $T$ is also a Fredholm operator with zero index. Thus, we only need to prove injectivity. In fact, if $\widehat{S} \psi-a=0$ and $\int_{\Gamma} \psi(x) d x=0$, then $\langle\psi, \widehat{S} \psi\rangle=0$. The inequality (4.1) implies that $\psi=0$ and consequently $a=0$. Thus $T$ is invertible. 
Let $\psi_{e}=\left(\psi_{1}, \psi_{2}\right)$ and $a_{e}=\left(a_{1}, a_{2}\right)$ be the unique solution to the following system

$$
T\left(\psi_{e}, a_{e}\right)=\left(0, e_{1}\right),
$$

Let $\tilde{\psi}_{e}=\left(\psi_{2}(-x), \psi_{1}(-x)\right)$ and $\tilde{a}_{e}=\left(a_{2}, a_{1}\right)$. By taking into account the symmetries of the kernel of $\widehat{S}$ we obtain

$$
T\left(\tilde{\psi}_{e}, \tilde{a}_{e}\right)=\left(0, e_{2}\right) .
$$

Theorem 4.5. Let $\delta_{1}$ be a fixed real constant such that $\delta_{1} \neq-a_{1} \pm a_{2}$. Then, $\mathcal{S}$ is invertible from $\left[\widetilde{H}^{-1 / 2}(\Gamma)\right]^{2}$ to $\left[H^{1 / 2}(\Gamma)\right]^{2}$.

Proof. It follows from the definition of $\widehat{S}$ that $\mathcal{S}=\delta_{1}\left(\begin{array}{l}\left\langle., e_{1}\right\rangle \\ \left\langle., e_{2}\right\rangle\end{array}\right)+\widehat{S}$. Lemma 4.4 implies that $\mathcal{S}$ is of Fredholm type with index zero. Thus, we only need to prove injectivity. Assume that $\mathcal{S} \varphi=0$ with $\varphi \in\left[\widetilde{H}^{-1 / 2}(\Gamma)\right]^{2}$. Let

Then, $\varphi_{0}$ belongs to $\left[\widetilde{H}_{0}^{-1 / 2}(\Gamma)\right]^{2}$ and satisfies

$$
\varphi_{0}:=\varphi-\left\langle\varphi, e_{1}\right\rangle \psi_{e}-\left\langle\varphi, e_{2}\right\rangle \tilde{\psi}_{e} .
$$

$$
\widehat{S} \varphi_{0}=-\left(\begin{array}{ll}
\delta_{1}+a_{1} & a_{2} \\
a_{2} & \delta_{1}+a_{1}
\end{array}\right) \int_{\Gamma} \varphi(x) d x .
$$

Consequently $\left\langle\varphi_{0}, \widehat{S} \varphi_{0}\right\rangle=0$. We deduce from Lemma 4.3 that $\varphi_{0}=0$ and hence $\varphi$ is also zero.

Remark 4.6. We assume throughout the paper that $\delta_{1} \in \mathbb{R}$ is fixed such that $\delta_{1} \neq-a_{1} \pm a_{2}$. The constant $\delta_{2}$ is immediately determined by the relation $\delta_{1}+\delta_{2}=\delta$, where $\delta$ is defined by (3.8). Notice that the constants $\delta_{i}, i=1,2$ are independent of $w$.

Define

$$
\mathcal{L}_{w}(k):=\mathcal{S}+\mathcal{R}_{w}(k)
$$

By (3.19) and Theorem 4.5, the operator $\mathcal{L}_{w}(k)$ is invertible from $\left[\widetilde{H}^{-1 / 2}(\Gamma)\right]^{2}$ to $\left[H^{1 / 2}(\Gamma)\right]^{2}$ for $w$ small enough (depending only on $w_{0}$ ), and it admits the following asymptotic expansion

$$
\begin{aligned}
\mathcal{L}_{w}^{-1}(k)= & \mathcal{S}^{-1} \sum_{m=0}^{+\infty}\left(-\mathcal{R}_{w}(k) \mathcal{S}^{-1}\right)^{m} \\
= & \mathcal{S}^{-1}-\mathcal{S}^{-1} \mathcal{S}_{1} \mathcal{S}^{-1} w-\mathcal{S}^{-1} \mathcal{T}_{2}(k) \mathcal{S}^{-1} w^{2} \ln w+\left[\mathcal{S}^{-1} \mathcal{S}_{1}^{2} \mathcal{S}^{-2}-\mathcal{S}^{-1} \mathcal{S}_{2}(k) \mathcal{S}^{-1}\right] w^{2} \\
& +2 \mathcal{S}^{-1} \mathcal{S}_{1} \mathcal{T}_{2}(k) \mathcal{S}^{-2} w^{3} \ln w+\left[2 \mathcal{S}^{-1} \mathcal{S}_{1} \mathcal{S}_{2}(k) \mathcal{S}^{-2}-\mathcal{S}^{-1} \mathcal{S}_{1}^{3} \mathcal{S}^{-3}-\mathcal{S}^{-1} \mathcal{S}_{3}(k) \mathcal{S}^{-1}\right] w^{3} \\
& +\sum_{\substack{n \geq 4 \\
m \geq 0}} \mathcal{L}_{m n}(k) w^{n}(\ln w)^{m},
\end{aligned}
$$

where each $\mathcal{L}_{m n}(k)$ is a compact operator from $\left[H^{1 / 2}(\Gamma)\right]^{2}$ to $\left[\widetilde{H}^{-1 / 2}(\Gamma)\right]^{2}$, holomorphic with respect to $k \in D_{r_{0}}^{+}$. Moreover, since $\rho_{w}(x, y, \cdot)$ defined by $(3.18)$, is analytic in $D_{r_{0}}^{+}$for every $(x, y) \in \bar{\Gamma} \times \bar{\Gamma}$, it follows that the kernel of $\mathcal{L}_{w}(k)$ is analytic with respect to $k \in D_{r_{0}}^{+}$, and that the operator valued function $k \mapsto \mathcal{L}_{w}(k)$ is holomorphic in the domain $D_{r_{0}}^{+}$. As a consequence of the Steinberg Theorem (Theorem 6.3), we deduce that $k \mapsto \mathcal{L}_{w}^{-1}(k)$ is holomorphic as well in the same domain $D_{r_{0}}^{+}$.

Remark 4.7. Let $\phi$ and $\psi \in\left[\widetilde{H}^{-1 / 2}(\Gamma)\right]^{2}$ be such that $\mathcal{L}_{w}(k) \phi=e_{1}$ and $\mathcal{L}_{w}(k) \psi=e_{2}$. Using the expression of the kernel of $\mathcal{L}_{w}(k)$ together with the fact that for each $n \geq 1$ the function $h_{n}(\cdot, k)$ in (3.15) is 4-periodic, we infer that $\phi_{1}(x)=\psi_{2}(-x)$ and consequently

$$
\left\langle\mathcal{L}_{w}^{-1}(k) e_{1}, e_{1}\right\rangle=\left\langle\phi, e_{1}\right\rangle=\left\langle\phi_{1}, 1\right\rangle=\left\langle\psi_{2}, 1\right\rangle=\left\langle\boldsymbol{\psi}, e_{2}\right\rangle=\left\langle\mathcal{L}_{w}^{-1}(k) e_{2}, e_{2}\right\rangle .
$$

Moreover, since $\mathcal{L}_{w}(k)$ is a self adjoint operator from $\left[\widetilde{H}^{-1 / 2}(\Gamma)\right]^{2}$ to $\left[H^{1 / 2}(\Gamma)\right]^{2}$, it follows that

$$
\left\langle\mathcal{L}_{w}^{-1}(k) e_{1}, e_{2}\right\rangle=\left\langle\mathcal{L}_{w}^{-1}(k) e_{2}, e_{1}\right\rangle .
$$


Remark 4.8. We define the matrix

$$
Q_{w}(k):=\left(\begin{array}{cc}
\left\langle e_{1}, \mathcal{L}_{w}^{-1}(k) e_{1}\right\rangle & \left\langle e_{1}, \mathcal{L}_{w}^{-1}(k) e_{2}\right\rangle \\
\left\langle e_{1}, \mathcal{L}_{w}^{-1}(k) e_{2}\right\rangle & \left\langle e_{1}, \mathcal{L}_{w}^{-1}(k) e_{1}\right\rangle
\end{array}\right)
$$

By the previous argument, the mapping $k \mapsto Q_{w}(k)$ is holomorphic, and using the asymptotic expansion (4.11) of $\mathcal{L}_{w}^{-1}(k)$, one can also expand $Q_{w}(k)$ as

$$
Q_{w}(k)=Q_{0}-Q_{1} w-Q_{12}(k) w^{2} \ln w+Q_{2}(k) w^{2}+Q_{13}(k) w^{3} \ln w+Q_{3}(k) w^{3}+\sum_{\substack{n \geq 4 \\ m \geq 0}} Q_{m n}(k) w^{n}(\ln w)^{m},
$$

where

$$
Q_{0}=\left(\begin{array}{cc}
\left\langle e_{1}, \mathcal{S}^{-1} e_{1}\right\rangle & \left\langle e_{1}, \mathcal{S}^{-1} e_{2}\right\rangle \\
\left\langle e_{1}, \mathcal{S}^{-1} e_{2}\right\rangle & \left\langle e_{1}, \mathcal{S}^{-1} e_{1}\right\rangle
\end{array}\right), \quad Q_{1}=\left(\begin{array}{cc}
\left\langle e_{1}, \mathcal{S}^{-1} \mathcal{S}_{1} \mathcal{S}^{-1} e_{1}\right\rangle & \left\langle e_{1}, \mathcal{S}^{-1} \mathcal{S}_{1} \mathcal{S}^{-1} e_{2}\right\rangle \\
\left\langle e_{1}, \mathcal{S}^{-1} \mathcal{S}_{1} \mathcal{S}^{-1} e_{2}\right\rangle & \left\langle e_{1}, \mathcal{S}^{-1} \mathcal{S}_{1} \mathcal{S}^{-1} e_{1}\right\rangle
\end{array}\right)
$$

and $Q_{12}(k), Q_{2}(k), Q_{13}(k), Q_{3}(k)$ and $Q_{m n}(k)$ (for $n \geq 4, m \geq 0$ ) are $2 \times 2$ matrices which are holomorphic with respect to $k$.

Let $\varphi_{i}:=\mathcal{S}^{-1} e_{i}, i=1,2$. A straight-forward computation implies that

$$
\left(\begin{array}{l}
\left\langle\psi_{e}, \mathcal{S} \varphi_{i}\right\rangle \\
\left\langle\tilde{\psi}_{e}, \mathcal{S} \varphi_{i}\right\rangle
\end{array}\right)=\left(\begin{array}{ll}
\delta_{1}+a_{1} & a_{2} \\
a_{2} & \delta_{1}+a_{1}
\end{array}\right) \int_{\Gamma} \varphi_{i}(x) d x=e_{i} \quad i=1,2 .
$$

Note that thanks to Remark 4.6, the matrix on the right hand-side is invertible and we have

$$
\left\langle e_{1}+e_{2}, \varphi_{i}\left\langle=\int_{\Gamma} \varphi_{i}(x) d x=\lambda_{0}\left(\begin{array}{cc}
\delta_{1}+a_{1} & -a_{2} \\
-a_{2} & \delta_{1}+a_{1}
\end{array}\right) e_{i} \quad i=1,2,\right.\right.
$$

where $\lambda_{0}=\left(\left(\delta_{1}+a_{1}\right)^{2}-a_{2}^{2}\right)^{-1}$. It follows that

$$
\operatorname{det} Q_{0}=\lambda_{0} \neq 0
$$

and thus, the matrix $Q_{0}$ is invertible. In the sequel, we also consider the following quantities

and

$$
\begin{gathered}
q_{0}^{ \pm}:=\left\langle e_{1}, \mathcal{S}^{-1}\left(e_{1} \pm e_{2}\right)\right\rangle, \quad q_{1}^{ \pm}:=\left\langle e_{1}, \mathcal{S}^{-1} \mathcal{S}_{1} \mathcal{S}^{-1}\left(e_{1} \pm e_{2}\right)\right\rangle, \\
q_{12}^{ \pm}(k):=\left\langle e_{1}, \mathcal{S}^{-1} \mathcal{T}_{2}(k) \mathcal{S}^{-1}\left(e_{1} \pm e_{2}\right)\right\rangle, \\
q_{2}^{ \pm}(k):=\left\langle e_{1},\left(\mathcal{S}^{-1} \mathcal{S}_{1}^{2} \mathcal{S}^{-2}-\mathcal{S}^{-1} \mathcal{S}_{2}(k) \mathcal{S}^{-1}\right)\left(e_{1} \pm e_{2}\right)\right\rangle, \\
q_{13}^{ \pm}(k):=2\left\langle e_{1}, \mathcal{S}^{-1} \mathcal{S}_{1} \mathcal{T}_{2}(k) \mathcal{S}^{-2}\left(e_{1} \pm e_{2}\right)\right\rangle
\end{gathered}
$$

$$
q_{3}^{ \pm}(k):=\left\langle e_{1},\left(2 \mathcal{S}^{-1} \mathcal{S}_{1} \mathcal{S}_{2}(k) \mathcal{S}^{-2}-\mathcal{S}^{-1} \mathcal{S}_{1}^{3} \mathcal{S}^{-3}-\mathcal{S}^{-1} \mathcal{S}_{3}(k) \mathcal{S}^{-1}\right)\left(e_{1} \pm e_{2}\right)\right\rangle
$$

Note that according to the definition of the kernels of the operators $\mathcal{S}, \mathcal{S}_{1}, \mathcal{S}_{n}(k)$ and $\mathcal{T}_{n}(k)$ for $n \geq 2$, all the above quantities are real numbers except for $q_{2}^{ \pm}(k)$ and $q_{3}^{ \pm}(k)$ (see (3.4) and (3.7)).

We now derive an explicit expression for the inverse of $\mathcal{S}_{w}(k)$.

Theorem 4.9. For $w \in\left(0, w_{0}\right)$ small enough and $k \in D_{r_{0}}^{+}$,

$$
\mathcal{S}_{w}^{-1}(k)=\mathcal{L}_{w}^{-1}(k)-\mathcal{L}_{w}^{-1}(k) \theta_{w}(k) F_{w}^{-1}(k)\left(\begin{array}{c}
\left\langle\cdot, \mathcal{L}_{w}^{-1}(k) e_{1}\right\rangle \\
\left\langle\cdot, \mathcal{L}_{w}^{-1}(k) e_{2}\right\rangle
\end{array}\right),
$$

where $F_{w}(k):=\mathrm{I}+\theta_{w}(k) Q_{w}(k), \theta_{w}(k)$ is given by $(3.2)$, and $Q_{w}(k)$ is defined in (4.12). Moreover, the resonances of the open cavities coincide with the poles of the matrix valued function $k \mapsto F_{w}^{-1}(k)$.

Proof. Let $\phi \in\left[\widetilde{H}^{-1 / 2}(\Gamma)\right]^{2}$ and $g \in\left[H^{1 / 2}(\Gamma)\right]^{2}$ be such that $\mathcal{S}_{w}(k) \phi=\left(\boldsymbol{\Theta}_{w}(k)+\mathcal{L}_{w}(k)\right) \phi=g$. Applying the operator $\mathcal{L}_{w}^{-1}(k)$ on the left we obtain that

$$
\phi=\mathcal{L}_{w}^{-1}(k) g-\mathcal{L}_{w}^{-1}(k) \theta_{w}(k)\left(\begin{array}{c}
\left\langle\phi, e_{1}\right\rangle \\
\left\langle\phi, e_{2}\right\rangle
\end{array}\right) .
$$




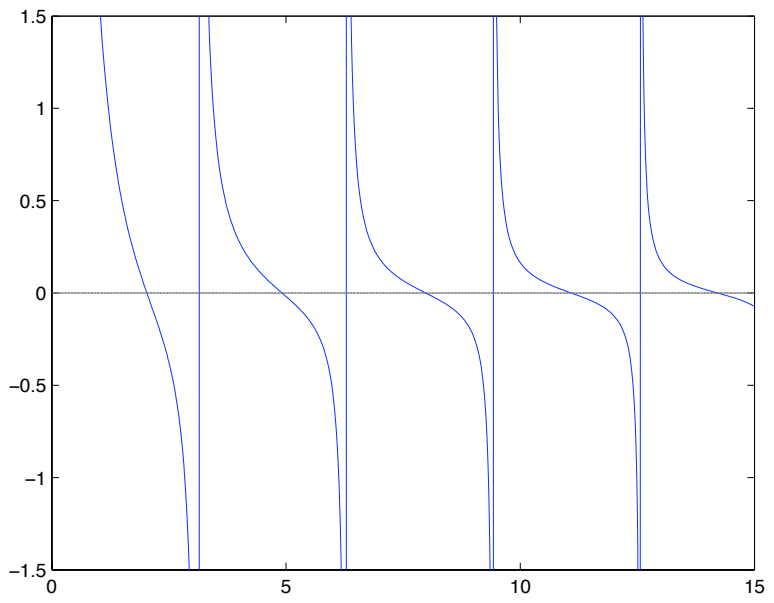

Figure 1. The function $k \mapsto \alpha(k)$

Taking the scalar product of (4.14) with $e_{i} \in\left[H^{1 / 2}(\Gamma)\right]^{2}(i=1$ and 2$)$ and using the fact that $\mathcal{L}_{w}^{-1}(k)$ is a symmetric operator, we find

$$
\left\langle\theta_{w}(k)\left(\begin{array}{c}
\left\langle\phi, e_{1}\right\rangle \\
\left\langle\phi, e_{2}\right\rangle
\end{array}\right), \mathcal{L}_{w}^{-1}(k) e_{i}\right\rangle+\left\langle\phi, e_{i}\right\rangle=\left\langle g, \mathcal{L}_{w}^{-1}(k) e_{i}\right\rangle .
$$

Decomposing the matrix $\theta_{w}(k)$ on the basis $\left\{e_{i} \otimes e_{j}\right\}_{i, j=1,2}$ we obtain the following system

$$
\left(\mathrm{I}+\theta_{w}(k) Q_{w}(k)\right)\left(\begin{array}{c}
\left\langle\phi, e_{1}\right\rangle \\
\left\langle\phi, e_{2}\right\rangle
\end{array}\right)=F_{w}(k)\left(\begin{array}{c}
\left\langle\phi, e_{1}\right\rangle \\
\left\langle\phi, e_{2}\right\rangle
\end{array}\right)=\left(\begin{array}{c}
\left\langle g, \mathcal{L}_{w}^{-1}(k) e_{1}\right\rangle \\
\left\langle g, \mathcal{L}_{w}^{-1}(k) e_{2}\right\rangle
\end{array}\right) .
$$

This system is invertible provided that $\operatorname{det} F_{w}(k) \neq 0$, in which case we deduce

$$
\left(\begin{array}{c}
\left\langle\phi, e_{1}\right\rangle \\
\left\langle\phi, e_{2}\right\rangle
\end{array}\right)=F_{w}^{-1}(k)\left(\begin{array}{c}
\left\langle g, \mathcal{L}_{w}^{-1}(k) e_{1}\right\rangle \\
\left\langle g, \mathcal{L}_{w}^{-1}(k) e_{2}\right\rangle
\end{array}\right)
$$

and the anounced expression (4.13) follows from (4.14) and (4.15). Finally, since the operator valued function $k \mapsto \mathcal{L}_{w}^{-1}(k)$ is holomorphic in $D_{r_{0}}^{+}$, it follows directly from (4.13) that the poles of $\mathcal{S}_{w}^{-1}(k)$ coincide with those of the matrix valued function $k \mapsto \theta_{w}(k) F_{w}^{-1}(k)$.

The poles of $\theta_{w}(k)$ are the set real values $\left\{\frac{n \pi}{h}\right\}_{n \in \mathbb{N}}$ and can not be the resonances of the open cavities. Since for a real frequency $k$, the problem (2.1) is well-posed. By a simple calculation one can verify that $\left\{\frac{n \pi}{h}\right\}_{n \in \mathbb{N}}$ are indeed not poles of $\theta_{w}(k) F_{w}^{-1}(k)$. Consequently, the resonances of the open cavities are the poles of $F_{w}^{-1}(k)$.

Let $\mathcal{Z}:=\left\{k_{\ell}\right\}_{\ell \in \mathbb{N}}$ be the set of zeros of the function $\alpha$ defined in (3.11), satisfying $k_{\ell}<k_{\ell+1}$ for all $\ell \in \mathbb{N}$. Let $\mathcal{P}:=\{\ell \pi / h\}_{\ell \in \mathbb{N}^{*}}$ denote the poles of $\alpha$. It is easily seen that each zero has multiplicity one. Moreover, since for each $\ell \in \mathbb{N}, k_{\ell}$ is an isolated point, there exists $r_{\ell}>0$ such that

$$
(\mathcal{Z} \cup \mathcal{P}) \cap D_{r_{\ell}}\left(k_{\ell}\right)=\left\{k_{\ell}\right\}
$$

We also introduce the integer $\ell_{0}:=\max \left\{\ell \in \mathbb{N}:\left|k_{\ell}\right|<r_{0}\right\}$.

We now prove the existence of two resonances close to each $k_{\ell}$, for every $\ell \in \mathbb{N}$.

Theorem 4.10. Let $w \in\left(0, w_{0}\right)$ and $k_{\ell} \in D_{r_{0}}^{+}$be a fixed zero of the function $\alpha$. Then, there exist two resonances of the open cavities $k_{\ell, w}^{+}$and $k_{\ell, w}^{-}$inside the disk $D_{r_{\ell}}\left(k_{\ell}\right)$ which are respectively the zeros of the functions

$$
\lambda_{w}^{+}(k):=1+\left[\frac{\alpha(k)}{w}+\frac{2}{\pi}(\ln k+\ln w)+2 \delta-\delta_{1}\right]\left\langle e_{1}, \mathcal{L}_{w}^{-1}(k)\left(e_{1}+e_{2}\right)\right\rangle,
$$


and

$$
\lambda_{w}^{-}(k):=1+\left(\frac{\alpha(k)}{w}-\delta_{1}\right)\left\langle e_{1}, \mathcal{L}_{w}^{-1}(k)\left(e_{1}-e_{2}\right)\right\rangle .
$$

Proof. Fix $\ell \in \mathbb{N}$. From the definition of $F_{w}(k), \theta_{w}(k)$ and $Q_{w}(k)$, we infer that for every $k \in \partial D_{r_{\ell}}\left(k_{\ell}\right)$,

and

$$
\begin{gathered}
F_{w}(k)=\frac{1}{w}\left[\alpha(k) Q_{0}+\Sigma_{w}(k)\right], \\
\frac{d F_{w}(k)}{d k}=\frac{1}{w}\left[\alpha^{\prime}(k) Q_{0}+\Sigma_{w}^{\prime}(k)\right],
\end{gathered}
$$

$$
F_{w}^{-1}(k)=\frac{w}{\alpha(k)} Q_{0}^{-1}\left[\mathrm{I}+\Upsilon_{w}(k)\right]
$$

where $\Sigma_{w}(k)$ and $\Upsilon_{w}(k)$ are $2 \times 2$ matrices which are holomorphic on $\partial D_{r_{\ell}}\left(k_{\ell}\right)$ and such that

$$
\sup _{k \in \partial D_{r_{\ell}}\left(k_{\ell}\right)}\left|\Sigma_{w}(k)\right| \rightarrow 0, \quad \sup _{k \in \partial D_{r_{\ell}}\left(k_{\ell}\right)}\left|\Sigma_{w}^{\prime}(k)\right| \rightarrow 0 \quad \text { and } \quad \sup _{k \in \partial D_{r_{\ell}}\left(k_{\ell}\right)}\left|\Upsilon_{w}(k)\right| \rightarrow 0
$$

as $w \rightarrow 0$. Therefore, we see that

$$
F_{w}^{-1}(k) \frac{d F_{w}(k)}{d k} \underset{w \rightarrow 0}{\longrightarrow} \frac{\alpha^{\prime}(k)}{\alpha(k)} \mathrm{I}
$$

uniformly with respect to $k \in \partial D_{r_{\ell}}\left(k_{\ell}\right)$, and thus

$$
\lim _{w \rightarrow 0} \operatorname{tr} \int_{\partial D_{r_{\ell}}\left(k_{\ell}\right)} F_{w}^{-1}(k) \frac{d F_{w}(k)}{d k} d k=2 \int_{\partial D_{r_{\ell}}\left(k_{\ell}\right)} \frac{\alpha^{\prime}(k)}{\alpha(k)} d k .
$$

On the other hand, by our choice of the radius $r_{\ell}$, we know that the function $\alpha$ admits exactly one zero and no pole inside the disk $D_{r_{\ell}}\left(k_{\ell}\right)$. Applying the Residue Theorem (see e.g. [19]) we deduce that

$$
\frac{1}{2 i \pi} \int_{\partial D_{r_{\ell}}\left(k_{\ell}\right)} \frac{\alpha^{\prime}(k)}{\alpha(k)} d k=1,
$$

and thus for $w$ small enough (depending on $\ell$ ) one has

$$
\frac{1}{2 i \pi} \operatorname{tr} \int_{\partial D_{r_{\ell}}\left(k_{\ell}\right)} F_{w}^{-1}(k) \frac{d F_{w}(k)}{d k} d k=2 .
$$

Thus the operator $\mathcal{S}_{w}(k)$ admits two characteristic values $k_{\ell, w}^{+}$and $k_{\ell, w}^{-}$in $D_{r_{\ell}}\left(k_{\ell}\right)$. A simple computation shows that the eigenvalues of $F_{w}(k)$ are given by the functions $\lambda_{w}^{ \pm}(k)$ defined in (4.16) and (4.17), and associated eigenvectors are $\frac{e_{1} \pm e_{2}}{\sqrt{2}}$. Thus, one can write

$$
F_{w}^{-1}(k)=\frac{\left(e_{1}+e_{2}\right) \otimes\left(e_{1}+e_{2}\right)}{2 \lambda_{w}^{+}(k)}+\frac{\left(e_{1}-e_{2}\right) \otimes\left(e_{1}-e_{2}\right)}{2 \lambda_{w}^{-}(k)},
$$

and consequently, the poles of $F_{w}^{-1}(k)$ in $D_{r_{\ell}}\left(k_{\ell}\right)$ are exactly the zeros of $\lambda_{w}^{ \pm}(k)$ in $D_{r_{\ell}}\left(k_{\ell}\right)$.

We next establish an asymptotic expansion of the resonances of the open cavities as their width $w$ tends to zero.

Theorem 4.11. For every $\ell \in \mathbb{N}$, the resonances $k_{\ell, w}^{ \pm}$have the following asymptotic expansion:

$$
\begin{aligned}
k_{\ell, w}^{+}= & k_{\ell}-\frac{2 w \ln w}{\pi \alpha^{\prime}\left(k_{\ell}\right)}-\frac{1}{\alpha^{\prime}\left(k_{\ell}\right)}\left[\frac{1}{q_{0}^{+}}+2\left(\frac{\ln k_{\ell}}{\pi}+\delta-\frac{\delta_{1}}{2}\right)\right] w+o(w), \\
k_{\ell, w}^{-}= & k_{\ell}-\frac{1-\delta_{1} q_{0}^{-}}{q_{0}^{-} \alpha^{\prime}\left(k_{\ell}\right)} w-\frac{1}{\left(q_{0}^{-}\right)^{2} \alpha^{\prime}\left(k_{\ell}\right)}\left[\frac{\left(1-\delta_{1} q_{0}^{-}\right)^{2} \alpha^{\prime \prime}\left(k_{\ell}\right)}{2 \alpha^{\prime}\left(k_{\ell}\right)^{2}}+q_{1}^{-}\right] w^{2}+\frac{q_{12}^{-}\left(k_{\ell}\right)}{\left(q_{0}^{-}\right)^{2} \alpha^{\prime}\left(k_{\ell}\right)} w^{3} \ln w \\
& +\frac{1}{\left(q_{0}^{-}\right)^{2} \alpha^{\prime}\left(k_{\ell}\right)}\left[q_{2}^{-}\left(k_{\ell}\right)-\frac{\left(q_{1}^{-}\right)^{2}}{q_{0}^{-}}-\frac{q_{1}^{-}\left(1-\delta_{1} q_{0}^{-}\right) \alpha^{\prime \prime}\left(k_{\ell}\right)}{q_{0}^{-} \alpha^{\prime}\left(k_{\ell}\right)^{2}}\right. \\
& \left.-\frac{\left(1-\delta_{1} q_{0}^{-}\right)^{3}}{2 q_{0}^{-} \alpha^{\prime}\left(k_{\ell}\right)^{3}}\left(\frac{\alpha^{\prime \prime}\left(k_{\ell}\right)^{2}}{\alpha^{\prime}\left(k_{\ell}\right)}-\frac{\alpha^{(3)}\left(k_{\ell}\right)}{3}\right)\right] w^{3}+o\left(w^{3}\right) .
\end{aligned}
$$


In particular, we have

$$
\begin{aligned}
\operatorname{Im}\left(k_{\ell, w}^{+}\right) & =\frac{w}{\alpha^{\prime}\left(k_{\ell}\right)}+o(w), \\
\operatorname{Im}\left(k_{\ell, w}^{-}\right) & =\frac{\operatorname{Im}\left(q_{2}^{-}\left(k_{\ell}\right)\right)}{\left(q_{0}^{-}\right)^{2} \alpha^{\prime}\left(k_{\ell}\right)} w^{3}+o\left(w^{3}\right) .
\end{aligned}
$$

Proof. Fix $\ell \in \mathbb{N}$. Since $k_{\ell, w}^{ \pm}$is a simple zero of the function $\lambda_{w}^{ \pm}(k)$ in $D_{r_{\ell}}\left(k_{\ell}\right)$, we deduce from the Residue Theorem that

$$
k_{\ell, w}^{ \pm}-k_{\ell}=\frac{1}{2 i \pi} \int_{\partial D_{r_{\ell}}\left(k_{\ell}\right)} \frac{\frac{d \lambda_{w}^{ \pm}}{d k}(k)}{\lambda_{w}^{ \pm}(k)}\left(k-k_{\ell}\right) d k .
$$

We now derive an asymptotic expansion of the functions $\frac{d \lambda_{w}^{ \pm}}{d k}(k) / \lambda_{w}^{ \pm}(k)$ in terms of $w$. We start with $\lambda_{w}^{+}(k)$. From (4.16) and (4.11) we have that for every $k \in \partial D_{r_{\ell}}\left(k_{\ell}\right)$,

and

$$
\frac{d \lambda_{w}^{+}}{d k}(k)=\frac{\alpha^{\prime}(k)}{w}\left\{q_{0}^{+}+\left[\frac{2 q_{0}^{+}}{k \pi \alpha^{\prime}(k)}-q_{1}^{+}\right] w+\varepsilon_{w, 1}^{+}(k)\right\}
$$

$$
\frac{1}{\lambda_{w}^{+}(k)}=\frac{w}{\alpha(k) q_{0}^{+}}\left\{1-\frac{2 w \ln w}{\pi \alpha(k)}-\left[\frac{2}{\alpha(k)}\left(\frac{\ln k}{\pi}+\delta-\frac{\delta_{1}}{2}\right)+\frac{1}{q_{0}^{+}}\left(\frac{1}{\alpha(k)}-q_{1}^{+}\right)\right] w+\varepsilon_{w, 2}^{+}(k)\right\},
$$

for some functions $\varepsilon_{w, 1}^{+}(k)$ and $\varepsilon_{w, 2}^{+}(k)$ holomorphic on $\partial D_{r_{\ell}}\left(k_{\ell}\right)$, such that

$$
\sup _{k \in \partial D_{r_{\ell}}\left(k_{\ell}\right)} w^{-1}\left|\varepsilon_{w, 1}^{+}(k)\right| \underset{w \rightarrow 0}{\longrightarrow} 0 \quad \text { and } \sup _{k \in \partial D_{r_{\ell}}\left(k_{\ell}\right)} w^{-1}\left|\varepsilon_{w, 2}^{+}(k)\right| \underset{w \rightarrow 0}{\longrightarrow} 0 .
$$

Hence, we obtain that

$$
\frac{\frac{d \lambda_{w}^{+}}{d k}(k)}{\lambda_{w}^{+}(k)}=\frac{\alpha^{\prime}(k)}{\alpha(k)}\left\{1-\frac{2 w \ln w}{\pi \alpha(k)}+\left[\frac{2}{\pi k \alpha^{\prime}(k)}-\frac{2}{\alpha(k)}\left(\frac{\ln k}{\pi}+\delta-\frac{\delta_{1}}{2}\right)-\frac{1}{\alpha(k) q_{0}^{+}}\right] w+\eta_{w}^{+}(k)\right\},
$$

where $\eta_{w}^{+}(k)$ is a holomorphic function on $\partial D_{r_{\ell}}\left(k_{\ell}\right)$ such that

$$
\sup _{k \in \partial D_{r_{\ell}}\left(k_{\ell}\right)} w^{-1}\left|\eta_{w}^{+}(k)\right| \underset{w \rightarrow 0}{\longrightarrow} 0 .
$$

Since $k_{\ell}$ is a simple zero of the function $\alpha$, we deduce from the Residue Theorem that for every holomorphic function $f$ in $\overline{D_{r_{\ell}}\left(k_{\ell}\right)}$,

$$
\frac{1}{2 i \pi} \int_{\partial D_{r_{\ell}}\left(k_{\ell}\right)}\left(k-k_{\ell}\right) \frac{\alpha^{\prime}(k)}{\alpha(k)} f(k) d k=0,
$$

and

$$
\frac{1}{2 i \pi} \int_{\partial D_{r_{\ell}}\left(k_{\ell}\right)}\left(k-k_{\ell}\right) \frac{\alpha^{\prime}(k)}{\alpha(k)^{2}} f(k) d k=\frac{f\left(k_{\ell}\right)}{\alpha^{\prime}\left(k_{\ell}\right)} .
$$

Thanks to (4.21) and (4.21) we get the asymptotic expansion of $k_{\ell, w}^{+}$multiplying (4.19) by $k-k_{\ell}$ and integrating over $\partial D_{r_{\ell}}\left(k_{\ell}\right)$. The imaginary part of $k_{\ell, w}^{+}$is obtained using the expression (3.8) of $\delta$.

We now treat the other resonance $k_{\ell, w}^{-}$. We will observe a posteriori that the imaginary part of $k_{\ell, w}^{-}$ scales (at least) like $w^{3}$. For this reason, we expand the integrand $\frac{d \lambda_{w}^{-}}{d k}(k) / \lambda_{w}^{-}(k)$ up to third order. From (4.17) and (4.11) we have for $k \in \partial D_{r_{\ell}}\left(k_{\ell}\right)$,

$$
\begin{aligned}
\frac{d \lambda_{w}^{-}}{d k}(k)= & \frac{\alpha^{\prime}(k) q_{0}^{-}}{w}\left\{1-\frac{q_{1}^{-}}{q_{0}^{-}} w+\frac{1}{q_{0}^{-}}\left[q_{12}^{-}(k)+\frac{\alpha(k)}{\alpha^{\prime}(k)} \frac{d q_{12}^{-}}{d k}(k)\right] w^{2} \ln w\right. \\
& +\frac{1}{q_{0}^{-}}\left[q_{2}^{-}(k)+\frac{\alpha(k)}{\alpha^{\prime}(k)} \frac{d q_{2}^{-}}{d k}(k)\right] w^{2} \\
& +\frac{1}{q_{0}^{-}}\left[q_{13}^{-}(k)+\frac{\alpha(k)}{\alpha^{\prime}(k)} \frac{d q_{13}^{-}}{d k}(k)-\frac{\delta_{1}}{\alpha^{\prime}(k)} \frac{d q_{12}^{-}}{d k}(k)\right] w^{3} \ln w \\
& \left.+\frac{1}{q_{0}^{-}}\left[q_{3}^{-}(k)+\frac{\alpha(k)}{\alpha^{\prime}(k)} \frac{d q_{3}^{-}}{d k}(k)-\frac{\delta_{1}}{\alpha^{\prime}(k)} \frac{d q_{2}^{-}}{d k}(k)\right] w^{3}+\varepsilon_{w, 1}^{-}(k)\right\}
\end{aligned}
$$


and

$$
\begin{aligned}
\frac{1}{\lambda_{w}^{-}(k)}= & \frac{w}{\alpha(k) q_{0}^{-}}\left\{1-\frac{1}{q_{0}^{-}}\left(\frac{1-\delta_{1} q_{0}^{-}}{\alpha(k)}-q_{1}^{-}\right) w-\frac{q_{12}^{-}(k)}{q_{0}^{-}} w^{2} \ln w\right. \\
+ & +\frac{1}{q_{0}^{-}}\left[\frac{1}{q_{0}^{-}}\left(\frac{1-\delta_{1} q_{0}^{-}}{\alpha(k)}-q_{1}^{-}\right)^{2}-\left(q_{2}^{-}(k)+\frac{\delta_{1} q_{1}^{-}}{\alpha(k)}\right)\right] w^{2} \\
+ & \frac{1}{q_{0}^{-}}\left[\frac{2 q_{12}^{-}(k)}{q_{0}^{-}}\left(\frac{1-\delta_{1} q_{0}^{-}}{\alpha(k)}-q_{1}^{-}\right)-\left(q_{13}^{-}(k)-\frac{\delta_{1} q_{12}^{-}}{\alpha(k)}\right)\right] w^{3} \ln w \\
+ & +\frac{1}{q_{0}^{-}}\left[\frac{2}{q_{0}^{-}}\left(\frac{1-\delta_{1} q_{0}^{-}}{\alpha(k)}-q_{1}^{-}\right)\left(q_{2}^{-}(k)+\frac{\delta_{1} q_{1}^{-}}{\alpha(k)}\right)-\frac{1}{\left(q_{0}^{-}\right)^{2}}\left(\frac{1-\delta_{1} q_{0}^{-}}{\alpha(k)}-q_{1}^{-}\right)^{3}\right. \\
& \left.\left.-\left(q_{3}^{-}(k)-\frac{\delta_{1} q_{2}^{-}}{\alpha(k)}\right)\right] w^{3}+\varepsilon_{w, 2}^{-}(k)\right\},
\end{aligned}
$$

for some holomorphic functions $\varepsilon_{w, 1}^{-}(k)$ and $\varepsilon_{w, 2}^{-}(k)$ defined on $\partial D_{r_{\ell}}\left(k_{\ell}\right)$, such that

$$
\sup _{k \in \partial D_{r_{\ell}}\left(k_{\ell}\right)} w^{-3}\left|\varepsilon_{w, 1}^{-}(k)\right| \underset{w \rightarrow 0}{\longrightarrow} 0 \text { and } \sup _{k \in \partial D_{r_{\ell}}\left(k_{\ell}\right)} w^{-3}\left|\varepsilon_{w, 2}^{-}(k)\right| \underset{w \rightarrow 0}{\longrightarrow} 0 .
$$

Hence, we obtain that

$$
\begin{aligned}
\frac{\frac{d \lambda_{w}^{-}}{d k}(k)}{\lambda_{w}^{-}(k)}= & \frac{\alpha^{\prime}(k)}{\alpha(k)}\left\{1-\frac{1-\delta_{1} q_{0}^{-}}{q_{0}^{-} \alpha(k)} w+\frac{\alpha(k)}{q_{0}^{-} \alpha^{\prime}(k)} \frac{d q_{12}^{-}}{d k}(k) w^{2} \ln w\right. \\
& +\frac{1}{q_{0}^{+}}\left[\frac{\alpha(k)}{\alpha^{\prime}(k)} \frac{d q_{2}^{-}}{d k}(k)-\frac{\delta_{1} q_{1}^{-}}{\alpha(k)}+\frac{1-\delta_{1} q_{0}^{-}}{q_{0}^{-} \alpha(k)}\left(\frac{1-\delta_{1} q_{0}^{-}}{\alpha(k)}-q_{1}^{-}\right)\right] w^{2} \\
& +\frac{1}{q_{0}^{+}}\left[\frac{q_{12}^{-}(k)}{q_{0}^{-} \alpha(k)}+\frac{\alpha(k)}{\alpha^{\prime}(k)}\left(\frac{d q_{13}^{-}}{d k}(k)-\frac{d q_{12}^{-}}{d k}(k) \frac{1}{q_{0}^{-}}\left(\frac{1}{\alpha(k)}-q_{1}^{-}\right)\right)\right] w^{3} \ln w \\
& +\frac{1}{q_{0}^{-}}\left[\frac{\alpha(k)}{\alpha^{\prime}(k)} \frac{d q_{3}^{-}}{d k}(k)-\frac{\alpha(k)}{\alpha^{\prime}(k) q_{0}^{-}} \frac{d q_{2}^{-}}{d k}(k)\left(\frac{1}{\alpha(k)}-q_{1}^{-}\right)+\frac{q_{2}^{-}(k)}{\alpha(k) q_{0}^{-}}+\frac{\delta_{1}\left(q_{1}^{-}\right)^{2}}{q_{0}^{-} \alpha(k)}\right. \\
& \left.\left.+\left(\frac{1-\delta_{1} q_{0}^{-}}{\alpha(k)}-q_{1}^{-}\right)\left(\frac{q_{1}^{-}\left(1+\delta_{1} q_{0}^{-}\right)}{\left(q_{0}^{-}\right)^{2} \alpha(k)}-\frac{\left(1-\delta_{1} q_{0}^{-}\right)^{2}}{\left(q_{0}^{-}\right)^{2} \alpha(k)^{2}}\right)\right] w^{3}+\eta_{w}^{-}(k) .\right\},
\end{aligned}
$$

where again $\eta_{w}^{-}(k)$ is a holomorphic function on $\partial D_{r_{\ell}}\left(k_{\ell}\right)$ such that

$$
\sup _{k \in \partial D_{r_{\ell}}\left(k_{\ell}\right)} w^{-3}\left|\eta_{w}^{-}(k)\right| \underset{w \rightarrow 0}{\longrightarrow} 0 .
$$

Note that in this expression, the terms which are factors of $\frac{\alpha^{\prime}(k)}{\alpha(k)^{3}}$ and $\frac{\alpha^{\prime}(k)}{\alpha(k)^{4}}$ are independant of $k$. Since $k_{\ell}$ is a simple zero of the function $\alpha$, we deduce from the Residue Theorem that

$$
\frac{1}{2 i \pi} \int_{\partial D_{r_{\ell}}\left(k_{\ell}\right)}\left(k-k_{\ell}\right) \frac{\alpha^{\prime}(k)}{\alpha(k)^{3}} d k=-\frac{\alpha^{\prime \prime}\left(k_{\ell}\right)}{2 \alpha^{\prime}\left(k_{\ell}\right)^{3}}
$$

and

$$
\frac{1}{2 i \pi} \int_{\partial D_{r_{\ell}}\left(k_{\ell}\right)}\left(k-k_{\ell}\right) \frac{\alpha^{\prime}(k)}{\alpha(k)^{4}} d k=\left(\frac{\alpha^{\prime \prime}\left(k_{\ell}\right)^{2}}{2 \alpha^{\prime}\left(k_{\ell}\right)^{5}}-\frac{\alpha^{(3)}\left(k_{\ell}\right)}{6 \alpha^{\prime}\left(k_{\ell}\right)^{4}}\right) .
$$

Thanks to (4.23), (4.24) we get the asymptotic expansion of $k_{\ell, w}^{-}$multiplying (4.22) by $k-k_{\ell}$ and integrating over $\partial D_{r_{\ell}}\left(k_{\ell}\right)$. The imaginary part of $k_{\ell, w}^{-}$is obtained noticing that the only complex number in the expression of $k_{\ell, w}^{-}$is $q_{2}^{-}\left(k_{\ell}\right)$ (see Remark 4.8).

Remark 4.12. Using the definition of $q_{2}^{-}\left(k_{\ell}\right)$ in Remark 4.8 together with (3.4), (3.7), (3.15) and the fact that $\mathcal{S}^{-1}$ is symmetric we infer that

$$
\operatorname{Im}\left(q_{2}^{-}\left(k_{\ell}\right)\right)=-\frac{\left\langle\mathcal{S}^{-1}\left(e_{1}-e_{2}\right), \mathcal{S}_{2}\left(k_{\ell}\right) \mathcal{S}^{-1}\left(e_{1}-e_{2}\right)\right\rangle}{2} .
$$


Moreover since the imaginary part of $\mathcal{S}_{2}\left(k_{\ell}\right)$ is an integral operator with kernel

$$
(x, y) \mapsto \frac{k_{\ell}^{2}}{8}\left(\begin{array}{cc}
(x-y)^{2} & (x-y+2 d)^{2} \\
(x-y-2 d)^{2} & (x-y)^{2}
\end{array}\right),
$$

we deduce, expanding the squares, that

$$
\operatorname{Im}\left(q_{2}^{-}\left(k_{\ell}\right)\right)=\frac{k_{\ell}^{2}}{8}\left(\left\langle x e_{1}+x e_{2}, \mathcal{S}^{-1}\left(e_{1}-e_{2}\right)\right\rangle-2 q_{0}^{-} d\right)^{2} .
$$

Since in view of (3.3) the operator $\mathcal{S}$ depends on $d$, it is possible to prove that the mapping $d \mapsto$ $\left\langle x e_{1}+x e_{2}, \mathcal{S}^{-1}\left(e_{1}-e_{2}\right)\right\rangle-2 q_{0}^{-} d$ is analytic in $(1,+\infty)$. Hence $\operatorname{Im}\left(q_{2}^{-}\left(k_{\ell}\right)\right)$ vanishes for at most countably many isolated points. Thus, either we choose $d>1$ so that $\operatorname{Im}\left(q_{2}^{-}\left(k_{\ell}\right)\right) \neq 0$ or one can perform a higher order asymptotic expansion to get a non zero coefficient that multiplies some power of $w$. Indeed, there exists $n \in \mathbb{N}(n \geq 3)$ such that $\operatorname{Im}\left(k_{\ell, w}^{-}\right)=c_{\ell}^{(n)} w^{n}+o\left(w^{n}\right)$ with $c_{\ell}^{(n)} \neq 0$ because, otherwise, the resonance $k_{\ell, w}^{-}$would be purely real. In any case, from the application point of view, what matters is that $\operatorname{Im}\left(k_{\ell}^{-}\right)<<\operatorname{Im}\left(k_{\ell}^{+}\right)$as $w$ tends to zero.

To conclude this section, we examine to behavior of the operator $\mathcal{S}_{w}^{-1}(k)$ in two different regions of the complex domain $D_{r_{0}}^{+}$. We first focus in the resonance zone where the contribution of the singular part is large with respect to the regular part. Close the resonances, we give an asymptotic expansion of $\mathcal{S}_{w}^{-1}(k)$ as a Laurent series, as well as an asymptotic expansion in terms of $w$.

Theorem 4.13. Let $k \in D_{r_{0}}^{+}$be a frequency close to the resonances. Then there exists a holomorphic operator $\mathcal{H}_{w}(k):\left[H^{1 / 2}(\Gamma)\right]^{2} \rightarrow\left[\widetilde{H}^{-1 / 2}(\Gamma)\right]^{2}$ and finite dimensional operators $\boldsymbol{\Delta}_{\ell, w}^{ \pm}:\left[H^{1 / 2}(\Gamma)\right]^{2} \rightarrow$ $\left[\widetilde{H}^{-1 / 2}(\Gamma)\right]^{2}\left(\right.$ for $\left.\ell=0, \ldots, \ell_{0}\right)$ such that

$$
\mathcal{S}_{w}^{-1}(k)=\sum_{\ell=0}^{\ell_{0}} \frac{\boldsymbol{\Delta}_{\ell, w}^{+}}{k-k_{\ell, w}^{+}}+\sum_{\ell=0}^{\ell_{0}} \frac{\boldsymbol{\Delta}_{\ell, w}^{-}}{k-k_{\ell, w}^{-}}+\mathcal{H}_{w}(k),
$$

where

$$
\boldsymbol{\Delta}_{\ell, w}^{ \pm}=-\frac{\left\langle\cdot, \mathcal{L}_{w}^{-1}\left(k_{\ell, w}^{ \pm}\right)\left(e_{1} \pm e_{2}\right)\right\rangle}{2 \frac{d \lambda_{w}^{ \pm}}{d k}\left(k_{\ell, w}^{ \pm}\right)} \mathcal{L}_{w}^{-1}\left(k_{\ell, w}^{ \pm}\right)\left[\theta_{w}\left(k_{\ell, w}^{ \pm}\right)\left(e_{1} \pm e_{2}\right)\right]
$$

Moreover, the following asymptotic expansions hold:

$$
\boldsymbol{\Delta}_{\ell, w}^{ \pm}=\frac{\left\langle\cdot, \mathcal{S}^{-1}\left(e_{1} \pm e_{2}\right)\right\rangle}{2\left(q_{0}^{ \pm}\right)^{2} \alpha^{\prime}\left(k_{\ell}\right)} \mathcal{S}^{-1}\left(e_{1} \pm e_{2}\right) w+\boldsymbol{\Lambda}_{\ell, w}^{ \pm},
$$

where $\boldsymbol{\Lambda}_{\ell, w}^{ \pm}$are compact operators from $\left[H^{1 / 2}(\Gamma)\right]^{2}$ to $\left[\widetilde{H}^{-1 / 2}(\Gamma)\right]^{2}$, satisfying

$$
w^{-1}\left\|\Lambda_{\ell, w}^{ \pm}\right\| \underset{w \rightarrow 0}{\longrightarrow} 0 \text {. }
$$

Proof. According to Theorem 4.2, Proposition 4.9 and Theorem 4.10, it is clear that $\mathcal{S}_{w}^{-1}(k)$ expands as $(4.25)$ close to the resonances, and that for each $\ell=0, \ldots, \ell_{0}$,

$$
\boldsymbol{\Delta}_{\ell, w}^{ \pm}=\lim _{k \rightarrow k_{\ell, w}^{ \pm}}\left(k-k_{\ell, w}^{ \pm}\right) \mathcal{S}_{w}^{-1}(k)
$$

Using the expression of $\mathcal{S}_{w}^{-1}(k)$ in $(4.13)$ and the fact that $\mathcal{L}_{w}^{-1}(k)$ and $\theta_{w}(k)$ are holomorphic in a neighborhood of $k_{\ell, w}^{ \pm}$, we infer that

$$
\begin{aligned}
\boldsymbol{\Delta}_{\ell, w}^{ \pm} & =-\mathcal{L}_{w}^{-1}\left(k_{\ell, w}^{ \pm}\right) \theta_{w}\left(k_{\ell, w}^{ \pm}\right)\left(e_{1} \pm e_{2}\right)\left\langle\cdot, \mathcal{L}_{w}^{-1}\left(k_{\ell, w}^{ \pm}\right)\left(e_{1} \pm e_{2}\right)\right\rangle \lim _{k \rightarrow k_{\ell, w}^{ \pm}} \frac{\left(k-k_{\ell, w}^{ \pm}\right)}{2 \lambda_{w}^{ \pm}(k)} \\
& =-\frac{\left\langle\cdot, \mathcal{L}_{w}^{-1}\left(k_{\ell, w}^{ \pm}\right)\left(e_{1} \pm e_{2}\right)\right\rangle}{2 \frac{d \lambda_{w}^{ \pm}}{d k}\left(k_{\ell, w}^{ \pm}\right)} \mathcal{L}_{w}^{-1}\left(k_{\ell, w}^{ \pm}\right)\left[\theta_{w}\left(k_{\ell, w}^{ \pm}\right)\left(e_{1} \pm e_{2}\right)\right],
\end{aligned}
$$

where we used expression (4.18) for $F_{w}^{-1}(k)$ in the first equality. 
We now derive the asymptotic expansion of $\boldsymbol{\Delta}_{\ell, w}^{-}$. According to (4.11) we have that

$$
\mathcal{L}_{w}^{-1}\left(k_{\ell, w}^{ \pm}\right)=\mathcal{S}^{-1}-\mathcal{S}^{-1} \mathcal{S}_{1} \mathcal{S}^{-1} w+\tilde{\mathbf{\Lambda}}_{\ell, w}^{ \pm}
$$

where $\tilde{\boldsymbol{\Lambda}}_{\ell, w}^{ \pm}$is a compact operator from $\left[H^{1 / 2}(\Gamma)\right]^{2}$ to $\left[\widetilde{H}^{-1 / 2}(\Gamma)\right]^{2}$ such that

$$
w^{-1}\left\|\tilde{\Lambda}_{\ell}^{ \pm}(w)\right\| \underset{w \rightarrow 0}{\longrightarrow} 0
$$

From (3.2), we have that

$$
\theta_{w}\left(k_{\ell, w}^{-}\right)\left(e_{1}-e_{2}\right)=\left(\frac{\alpha\left(k_{\ell, w}^{-}\right)}{w}-\delta_{1}\right)\left(e_{1}-e_{2}\right)
$$

and we use the Taylor expansion of $k \mapsto \alpha(k)$ around $k_{\ell}$ together with Theorem 4.11 to obtain

$$
\theta_{w}\left(k_{\ell, w}^{-}\right)\left(e_{1}-e_{2}\right)=\frac{1}{w}\left\{-\frac{w}{q_{0}^{-}}+o(w)\right\}\left(e_{1}-e_{2}\right) .
$$

Moreover, from (4.17) and (4.27), we deduce that

$$
\frac{d \lambda_{w}^{-}}{d k}\left(k_{\ell, w}^{-}\right)=\frac{\alpha^{\prime}\left(k_{\ell, w}^{-}\right) q_{0}^{-}}{w}\left(1-\frac{q_{1}^{-}}{q_{0}^{-}} w+o(w)\right)
$$

and using again a Taylor expansion of $k \mapsto \alpha^{\prime}(k)$ around $k_{\ell}$ together with Theorem 4.11 leads to

$$
\frac{d \lambda_{w}^{-}}{d k}\left(k_{\ell, w}^{-}\right)=\frac{\alpha^{\prime}\left(k_{\ell}\right) q_{0}^{-}}{w}\left\{1-\left[\frac{q_{1}^{-}}{q_{0}^{-}}+\frac{\left(1-\delta_{1} q_{0}^{-}\right) a^{\prime \prime}\left(k_{\ell}\right)}{q_{0}^{-} \alpha^{\prime}\left(k_{\ell}\right)^{2}}\right] w+o(w)\right\} .
$$

Hence, gathering (4.27), (4.28), (4.29) and (4.30), we obtain the expected asymptotic of $\boldsymbol{\Delta}_{\ell, w}^{-}$.

We proceed similarly for $\boldsymbol{\Delta}_{\ell, w}^{+}$. Indeed, from (3.2) we have

$$
\theta_{w}\left(k_{\ell, w}^{+}\right)\left(e_{1}+e_{2}\right)=\left\{\frac{\alpha\left(k_{\ell, w}^{+}\right)}{w}+\frac{2}{\pi}\left(\ln k_{\ell, w}^{+}+\ln w\right)+2 \delta-\delta_{1}\right\}\left(e_{1}+e_{2}\right),
$$

and thus, using a Taylor expansion of $k \mapsto \alpha(k)$ and $k \mapsto \ln k$ around $k_{\ell}$ together with Theorem 4.11, we see

$$
\theta_{w}\left(k_{\ell, w}^{+}\right)\left(e_{1}+e_{2}\right)=\frac{1}{w}\left\{-\frac{w}{q_{0}^{+}}+o(w)\right\}\left(e_{1}+e_{2}\right) .
$$

Moreover, from (4.16), we deduce

$$
\frac{d \lambda_{w}^{+}}{d k}\left(k_{\ell, w}^{+}\right)=\frac{1}{w}\left\{\alpha^{\prime}\left(k_{\ell, w}^{+}\right) q_{0}^{+}+\left[\frac{2 q_{0}^{+}}{k_{\ell, w}^{+} \pi}-q_{1}^{+} \alpha^{\prime}\left(k_{\ell, w}^{+}\right)\right] w+o(w)\right\},
$$

and a Taylor expansion of $k \mapsto \alpha^{\prime}(k)$ and $k \mapsto 1 / k$ around $k_{\ell}$ together with Theorem 4.11 leads to

$$
\begin{aligned}
\frac{d \lambda_{w}^{+}}{d k}\left(k_{\ell, w}^{+}\right)= & \frac{\alpha^{\prime}\left(k_{\ell}\right) q_{0}^{+}}{w}\left\{1-\frac{2 \alpha^{\prime \prime}\left(k_{\ell}\right)}{\pi \alpha^{\prime}\left(k_{\ell}\right)^{2}} w \ln w\right. \\
& \left.-\left[\frac{\alpha^{\prime \prime}\left(k_{\ell}\right)}{\alpha^{\prime}\left(k_{\ell}\right)^{2}}\left(\frac{1}{q_{0}^{+}}+2\left(\frac{\ln k_{\ell}}{\pi}+\delta-\frac{\delta_{1}}{2}\right)\right)-\frac{2}{\pi k_{\ell} \alpha^{\prime}\left(k_{\ell}\right)}+\frac{q_{1}^{+}}{q_{0}^{+}}\right] w+o(w)\right\} .
\end{aligned}
$$

Hence, gathering $(4.27),(4.31),(4.32)$ and $(4.33)$, we obtain the expected asymptotic of $\boldsymbol{\Delta}_{\ell, w}^{+}$.

In the non resonance zone, the contribution of the singular part is negligible. The following result gives an asymptotic expansion of $\mathcal{S}_{w}^{-1}(k)$ in terms of $w$ in this region $D_{r_{0}}^{+} \backslash \mathcal{Z}$, i.e., when $\alpha(k) \neq 0$. 
Theorem 4.14. If $k \in D_{r_{0}}^{+} \backslash \mathcal{Z}$, then

$$
\begin{aligned}
& \mathcal{S}_{w}^{-1}(k)=\mathcal{S}^{-1}-\frac{\mathcal{S}^{-1}\left(e_{1}+e_{2}\right)}{2 q_{0}^{+}}\left\langle\cdot, \mathcal{S}^{-1}\left(e_{1}+e_{2}\right)\right\rangle-\frac{\mathcal{S}^{-1}\left(e_{1}-e_{2}\right)}{2 q_{0}^{-}}\left\langle\cdot, \mathcal{S}^{-1}\left(e_{1}-e_{2}\right)\right\rangle \\
& +\left\{-\mathcal{S}^{-1} \mathcal{S}_{1} \mathcal{S}^{-1}+\left[\frac{\mathcal{S}^{-1}\left(e_{1}+e_{2}\right)}{2\left(q_{0}^{+}\right)^{2}}\left(\frac{1}{\alpha(k)}-q_{1}^{+}\right)+\frac{\mathcal{S}^{-1} \mathcal{S}_{1} \mathcal{S}^{-1}\left(e_{1}+e_{2}\right)}{2 q_{0}^{+}}\right]\left\langle\cdot, \mathcal{S}^{-1}\left(e_{1}+e_{2}\right)\right\rangle\right. \\
& +\left[\frac{\mathcal{S}^{-1}\left(e_{1}-e_{2}\right)}{2\left(q_{0}^{-}\right)^{2}}\left(\frac{1}{\alpha(k)}-q_{1}^{-}\right)+\frac{\mathcal{S}^{-1} \mathcal{S}_{1} \mathcal{S}^{-1}\left(e_{1}-e_{2}\right)}{2 q_{0}^{-}}\right]\left\langle\cdot, \mathcal{S}^{-1}\left(e_{1}-e_{2}\right)\right\rangle \\
& \left.+\frac{\mathcal{S}^{-1}\left(e_{1}+e_{2}\right)}{2 q_{0}^{+}}\left\langle\cdot, \mathcal{S}^{-1} \mathcal{S}_{1} \mathcal{S}^{-1}\left(e_{1}+e_{2}\right)\right\rangle+\frac{\mathcal{S}^{-1}\left(e_{1}-e_{2}\right)}{2 q_{0}^{-}}\left\langle\cdot, \mathcal{S}^{-1} \mathcal{S}_{1} \mathcal{S}^{-1}\left(e_{1}-e_{2}\right)\right\rangle\right\} w+\xi_{w}(k),
\end{aligned}
$$

where $\xi_{w}(k)$ is a holomorphic operator from $\left[H^{1 / 2}(\Gamma)\right]^{2}$ to $\left[\widetilde{H}^{-1 / 2}(\Gamma)\right]^{2}$ such that

$$
w^{-1}\left\|\xi_{w}(k)\right\| \underset{w \rightarrow 0}{\longrightarrow} 0 \quad \text { for every } k \in D_{r_{0}}^{+} \backslash \mathcal{Z} \text {. }
$$

Proof. Fix $k \in D_{r_{0}}^{+} \backslash \mathcal{Z}$. We use the expression (4.13) for $\mathcal{S}_{w}^{-1}(k)$ and (4.18) for $F_{w}^{-1}(k)$ to prove that

$$
\begin{aligned}
\mathcal{S}_{w}^{-1}(k)= & \mathcal{L}_{w}^{-1}(k)-\frac{\left\langle\cdot, \mathcal{L}_{w}^{-1}(k)\left(e_{1}-e_{2}\right)\right\rangle}{2 \lambda_{w}^{-}(k)} \mathcal{L}_{w}^{-1}(k) \theta_{w}(k)\left(e_{1}-e_{2}\right) \\
& -\frac{\left\langle\cdot, \mathcal{L}_{w}^{-1}(k)\left(e_{1}+e_{2}\right)\right\rangle}{2 \lambda_{w}^{+}(k)} \mathcal{L}_{w}^{-1}(k) \theta_{w}(k)\left(e_{1}+e_{2}\right) .
\end{aligned}
$$

Since $\alpha(k) \neq 0$, we get that

$$
\begin{aligned}
\frac{\left\langle\cdot, \mathcal{L}_{w}^{-1}(k)\left(e_{1}-e_{2}\right)\right\rangle}{2 \lambda_{w}^{-}(k)}= & \frac{w}{2 \alpha(k) q_{0}^{-}}\left\{\left\langle\cdot, \mathcal{S}^{-1}\left(e_{1}-e_{2}\right)\right\rangle+\left[\frac{1}{q_{0}^{-}}\left(q_{1}^{-}-\frac{1-\delta_{1} q_{0}^{-}}{\alpha(k)}\right)\left\langle\cdot, \mathcal{S}^{-1}\left(e_{1}-e_{2}\right)\right\rangle\right.\right. \\
& \left.\left.-\left\langle\cdot, \mathcal{S}^{-1} \mathcal{S}_{1} \mathcal{S}^{-1}\left(e_{1}-e_{2}\right)\right\rangle\right] w+\xi_{w}^{-}(k)\right\}
\end{aligned}
$$

and

$$
\begin{aligned}
\frac{\left\langle\cdot, \mathcal{L}_{w}^{-1}(k)\left(e_{1}+e_{2}\right)\right\rangle}{2 \lambda_{w}^{+}(k)}= & \frac{w}{2 \alpha(k) q_{0}^{+}}\left\{\left\langle\cdot, \mathcal{S}^{-1}\left(e_{1}+e_{2}\right)\right\rangle-\frac{2}{\pi \alpha(k)}\left\langle\cdot, \mathcal{S}^{-1}\left(e_{1}+e_{2}\right)\right\rangle w \ln w\right. \\
& -\left[\left(\frac{1}{q_{0}^{+}}\left(\frac{1}{\alpha(k)}-q_{1}^{+}\right)+\frac{2}{\alpha(k)}\left(\frac{\ln k}{\pi}+\delta-\frac{\delta_{1}}{2}\right)\right)\left\langle\cdot, \mathcal{S}^{-1}\left(e_{1}+e_{2}\right)\right\rangle\right. \\
& \left.\left.+\left\langle\cdot, \mathcal{S}^{-1} \mathcal{S}_{1} \mathcal{S}^{-1}\left(e_{1}+e_{2}\right)\right\rangle\right] w+\xi_{w}^{+}(k)\right\} .
\end{aligned}
$$

where $\xi_{w}^{ \pm}(k)$ are holomorphic operators from $\left[H^{1 / 2}(\Gamma)\right]^{2}$ to $\left[\widetilde{H}^{-1 / 2}(\Gamma)\right]^{2}$ such that

$$
w^{-1}\left\|\xi_{w}^{ \pm}(k)\right\| \underset{w \rightarrow 0}{\longrightarrow} 0 \quad \text { for every } k \in D_{r_{0}}^{+} \backslash \mathcal{Z}
$$

Formula (3.2) for $\theta_{w}(k)$ and the asymptotic expansion (4.11) of $\mathcal{L}_{w}^{-1}(k)$ complete the proof of the theorem.

\section{Asymptotic of the Field $u$}

We first derive an asymptotic expansion of the field $u(x ; w)$ far from the resonances, i.e., when $\alpha(k) \neq 0$. We distinguish the far field from the near field.

Theorem 5.1. Let $k \in D_{r_{0}}^{+} \backslash \mathcal{Z}$. Then for every $y \in \Omega_{e}$, one has

$$
u(y)=u_{e}(y)+\frac{i u_{e}(0)}{\alpha(k)} H_{0}^{(1)}(k|y|) w+\rho_{w}(y),
$$


for some function $\rho_{w} \in H_{\mathrm{loc}}^{1}\left(\Omega_{e}\right)$ such that $w^{-1} \rho_{w} \rightarrow 0$ in $H_{\mathrm{loc}}^{1}\left(\Omega_{e}\right)$. Moreover, if $y=\left(w y_{1} \pm w d, w y_{2}\right)$ for some $y_{1} \in \Gamma$ and $y_{2}>0$, then

$$
\begin{aligned}
u\left(w y_{1} \pm w d, w y_{2}\right)= & u_{e}\left(w y_{1} \pm w d, w y_{2}\right)-\frac{2 u_{e}(0)}{\pi \alpha(k)} w \ln w \\
& -\frac{2 u_{e}(0)}{\pi \alpha(k)}\left[\delta+\frac{\ln k}{\pi}+\frac{\ln \left|\left(y_{1} \pm d, y_{2}\right)\right|}{\pi}\right] w+\bar{\rho}_{w}\left(y_{1}, y_{2}\right),
\end{aligned}
$$

where $\bar{\rho}_{w} \in H^{1}\left(B_{1}^{+}\right)$satisfies $w^{-1} \bar{\rho}_{w} \rightarrow 0$ in $H^{1}\left(B_{1}^{+}\right)$.

Proof. Let $y \in \Omega_{e}$ be fixed. One has

$$
\begin{aligned}
G_{e}(w t, 0 ; y) & =-\frac{i}{2} H_{0}^{(1)}(k|y-(w t, 0)|) \\
& =-\frac{i}{2} H_{0}^{(1)}(k|y|)-\sum_{n=1}^{+\infty} \eta_{n}(y)(w t)^{n},
\end{aligned}
$$

uniformly with respect to $t \in[-4,4]$ and the functions $\eta_{n}(y)$ are in $C^{\infty}\left(\Omega_{e}\right)$. Using (2.4) we deduce

$$
\begin{aligned}
u_{e}(w t, 0) & =\int_{\Omega_{e}} G_{e}(w t, 0 ; z) f(z) d z \\
& =-\frac{i}{2} \int_{\Omega_{e}} H_{0}^{(1)}(k|z|) f(z) d z-\sum_{n=1}^{+\infty} \bar{\eta}_{n}(w t)^{n},
\end{aligned}
$$

uniformly with respect to $t \in[-4,4]$, where the coefficients $\bar{\eta}_{n}$ are defined by

$$
\bar{\eta}_{n}:=\int_{\Omega_{e}} \eta_{n}(y) f(y) d y .
$$

Using (2.7) together with the integral equations (2.9)-(2.10), and after a change of variables we get

$$
u(y)=u_{e}(y)-\left\langle\left(\begin{array}{c}
G_{e}(w \cdot-w d, 0 ; y) \\
G_{e}(w \cdot+w d, 0 ; y)
\end{array}\right), \mathcal{S}_{w}^{-1}(k)\left(\begin{array}{c}
u_{e}(w \cdot-w d, 0) \\
u_{e}(w \cdot+w d, 0)
\end{array}\right)\right\rangle .
$$

From Theorem 4.13 we have

$$
\mathcal{S}_{w}^{-1}(k)\left(e_{1} \pm e_{2}\right)=\frac{\mathcal{S}^{-1}\left(e_{1} \pm e_{2}\right)}{q_{0}^{ \pm} \alpha(k)} w+o(w) \quad \text { in }\left[\widetilde{H}^{-1 / 2}(\Gamma)\right]^{2},
$$

and thus the integral equations (2.9) and (2.10) imply

$$
\begin{aligned}
\mathcal{S}_{w}^{-1}(k)\left(\begin{array}{c}
u_{e}(w \cdot-w d, 0) \\
u_{e}(w \cdot+w d, 0)
\end{array}\right)= & -\frac{i}{2}\left(\int_{\Omega_{e}} H_{0}^{(1)}(k|z|) f(z) d z\right) \frac{\mathcal{S}^{-1}\left(e_{1}+e_{2}\right)}{q_{0}^{+} \alpha(k)} w \\
& -\bar{\eta}_{1} \mathcal{S}^{-1}\left(x e_{1}+x e_{2}\right) w \\
& +\bar{\eta}_{1} \frac{\mathcal{S}^{-1}\left(e_{1}+e_{2}\right)}{2 q_{0}^{+}}\left\langle\left(x e_{1}+x e_{2}\right), \mathcal{S}^{-1}\left(e_{1}+e_{2}\right)\right\rangle w \\
& +\bar{\eta}_{1} \frac{\mathcal{S}^{-1}\left(e_{1}-e_{2}\right)}{2 q_{0}^{-}}\left\langle\left(x e_{1}+x e_{2}\right), \mathcal{S}^{-1}\left(e_{1}-e_{2}\right)\right\rangle w+o(w)
\end{aligned}
$$

in $\left[\widetilde{H}^{-1 / 2}(\Gamma)\right]^{2}$. We now use (5.1) together with (5.4) to deduce

$$
\begin{aligned}
u(y)= & u_{e}(y) \\
& +\left(-\frac{i}{2} H_{0}^{(1)}(k|y|)+O(w)\right)\left\{\frac{i}{2}\left(\int_{\Omega_{e}} H_{0}^{(1)}(k|z|) f(z) d z\right) \frac{\left\langle\mathcal{S}^{-1}\left(e_{1}+e_{2}\right), e_{1}+e_{2}\right\rangle}{q_{0}^{+} \alpha(k)} w\right. \\
& +\bar{\eta}_{1}\left\langle\mathcal{S}^{-1}\left(x e_{1}+x e_{2}\right), e_{1}+e_{2}\right\rangle w \\
& -\bar{\eta}_{1} \frac{\left\langle\mathcal{S}^{-1}\left(e_{1}+e_{2}\right), e_{1}+e_{2}\right\rangle}{2 q_{0}^{+}}\left\langle\left(x e_{1}+x e_{2}\right), \mathcal{S}^{-1}\left(e_{1}+e_{2}\right)\right\rangle w \\
& \left.-\bar{\eta}_{1} \frac{\left\langle\mathcal{S}^{-1}\left(e_{1}-e_{2}\right), e_{1}+e_{2}\right\rangle}{2 q_{0}^{-}}\left\langle\left(x e_{1}+x e_{2}\right), \mathcal{S}^{-1}\left(e_{1}-e_{2}\right)\right\rangle w+o(w)\right\} .
\end{aligned}
$$


The terms $O(w)$ and $o(w)$ are uniform in $C_{l o c}^{1}\left(\Omega_{e}\right)$. In view of the symmetry of $\mathcal{S}^{-1}$, we have that $\left\langle\mathcal{S}^{-1}\left(e_{1}-e_{2}\right), e_{1}+e_{2}\right\rangle=0$, and consequently,

$$
u(y)=u_{e}(y)+\frac{H_{0}^{(1)}(k|y|)}{2 \alpha(k)}\left(\int_{\Omega_{e}} H_{0}^{(1)}(k|z|) f(z) d z\right) w+\rho_{w}(y),
$$

where $w^{-1} \rho_{w}(y) \rightarrow 0$ in $H_{\mathrm{loc}}^{1}\left(\Omega_{e}\right)$.

We now treat the asymptotic of the field $u$ close to the cavities. By (3.6), we know that for each $y \in \Omega_{e}$, we have that

$$
H_{0}^{(1)}\left(k\left|\left(w y_{1} \pm w d, w y_{2}\right)\right|\right)=\frac{2 i}{\pi} \ln w+2 i\left[\delta+\frac{\ln k}{\pi}+\frac{\ln \left|\left(y_{1} \pm d, y_{2}\right)\right|}{\pi}\right]+o(w) .
$$

This estimate holds pointwise and in $H^{1}\left(B_{1}^{+}\right)$as well. Then, we substitute the Green function $G_{e}(w$. $-w d, 0 ; y)$ by its asymptotic expansion (5.6) in (5.3) to complete the proof.

Remark 5.2. When the frequency $k \in D_{r_{0}}^{+} \backslash \mathcal{Z}$ is far from the resonances, the field $u$ behaves in a similar way to the case of a single cavity, studied in [8]. The asymptotic response is that of two independent cavities without interaction.

Remark 5.3. By $(5.1)$, it is clear that $\eta_{1}(y)=-\frac{i}{2} D_{x_{1}}\left[H_{0}^{(1)}(k|y|)\right]$ in the sense of distributions. Moreover, since the Hankel function $H_{0}^{(1)}$ has a logarithmic singularity at zero, the map $y \mapsto H_{0}^{(1)}(k|y|)$ belongs to $W_{\text {loc }}^{1, p}\left(\Omega_{e}\right)$ for any $p \in[1,2)$. Hence, if $f$ is more regular, e.g. $f \in W^{1, p^{\prime}}\left(\Omega_{e}\right)$, with $p^{\prime}=p /(p-1)$, with compact support in $\Omega_{e}$, then $u_{e}$ is more regular as well (in particular $u_{e} \in H_{\mathrm{loc}}^{3}\left(\Omega_{e}\right)$ ) and $\frac{\partial u_{e}}{\partial x_{1}}$ is a continuous function. It can be extended by continuity at the origin by setting

$$
\frac{\partial u_{e}}{\partial x_{1}}(0):=-\int_{\Omega_{e}} \eta_{1}(z) f(z) d z,
$$

We now derive an asymptotic expansion of the field $u$ close to the resonances $k_{\ell, w}^{ \pm}$, distinguishing again two regions of the plane: the far and the near fields.

Theorem 5.4. Let $k \in D_{r_{0}}^{+}$be a frequency close to the resonances.

1) Far field: for every $y \in \Omega_{e}$,

$$
u(y)=u_{e}(y)+\sum_{\ell=0}^{\ell_{0}} \frac{\kappa_{\ell, w}^{+}(y)}{k-k_{\ell, w}^{+}}+\sum_{\ell=0}^{\ell_{0}} \frac{\kappa_{\ell, w}^{-}(y)}{k-k_{\ell, w}^{-}}+U^{w}(k, y),
$$

where $k \mapsto U^{w}(k, y)$ is a holomorphic function in $D_{r_{0}}^{+}$for every $y \in \Omega_{e}$, and $U^{w}(k, \cdot) \in H_{\mathrm{loc}}^{1}\left(\Omega_{e}\right)$ for every $k \in D_{r_{0}}^{+}$. Moreover,

$$
\kappa_{\ell, w}^{+}(y)=\frac{i u_{e}(0)}{\alpha^{\prime}\left(k_{\ell}\right)} H_{0}^{(1)}\left(k_{\ell}|y|\right) w+\rho_{\ell, w}^{+}(y),
$$

and

$$
\kappa_{\ell, w}^{-}(y)=\frac{i}{4 \alpha^{\prime}\left(k_{\ell}\right)} \frac{\partial u_{e}}{\partial x_{1}}(0) D_{x_{1}}\left[H_{0}^{(1)}\left(k_{\ell}|y|\right)\right]\left(\frac{\left\langle x e_{1}+x e_{2}, \mathcal{S}^{-1}\left(e_{1}-e_{2}\right)\right\rangle}{q_{0}^{-}}-2 d\right)^{2} w^{3}+\rho_{\ell, w}^{-}(y),
$$

for some $\rho_{\ell, w}^{ \pm}(y) \in H_{\mathrm{loc}}^{1}\left(\Omega_{e}\right)$ such that $w^{-1} \rho_{\ell, w}^{+} \rightarrow 0$ and $w^{-3} \rho_{\ell, w}^{-} \rightarrow 0$ in $H_{\mathrm{loc}}^{1}\left(\Omega_{e}\right)$.

2) Near field: if further $y=\left(w y_{1} \pm w d, w y_{2}\right)$ for some $y_{1} \in \Gamma$ and $y_{2}>0$, the formula (5.7) is still valid and we have

$$
\begin{aligned}
\kappa_{\ell, w}^{+}\left(w y_{1} \pm w d, w y_{2}\right)= & -\frac{2 u_{e}(0)}{\pi \alpha^{\prime}\left(k_{\ell}\right)} w \ln w \\
& -\frac{2 u_{e}(0)}{\alpha^{\prime}\left(k_{\ell}\right)}\left(\delta+\frac{\ln k_{\ell}}{\pi}+\frac{\ln \left|\left(y_{1} \pm d, y_{2}\right)\right|}{\pi}\right) w+\bar{\rho}_{\ell, w}^{+}\left(y_{1}, y_{2}\right),
\end{aligned}
$$




$$
\begin{aligned}
\kappa_{\ell, w}^{-}\left(w y_{1}\right. & \left. \pm w d, w y_{2}\right) \\
=- & \frac{\left\langle\Gamma^{ \pm}\left(\cdot, y_{1}, y_{2}\right), \mathcal{S}^{-1}\left(e_{1}-e_{2}\right)\right\rangle}{2 \pi q_{0}^{-} \alpha^{\prime}\left(k_{\ell}\right)} \frac{\partial u_{e}}{\partial x_{1}}(0)\left(2 d-\frac{\left\langle x e_{2}+x e_{2}, \mathcal{S}^{-1}\left(e_{1}-e_{2}\right)\right\rangle}{q_{0}^{-}}\right) w^{2} \\
& +\bar{\rho}_{\ell, w}^{-}\left(y_{1}, y_{2}\right),
\end{aligned}
$$

where $\Gamma^{ \pm}$are defined in (5.8)-(5.9), and $\bar{\rho}_{\ell, w}^{ \pm} \in H^{1}\left(B_{1}^{+}\right)$satisfy $w^{-1} \bar{\rho}_{\ell, w}^{+} \rightarrow 0$ and $w^{-3} \bar{\rho}_{\ell, w}^{-} \rightarrow 0$ in $H^{1}\left(B_{1}^{+}\right)$.

Proof. From the integral equation (2.7) and Theorem 4.13, we deduce that expression (5.7) holds with

and

$$
\kappa_{\ell, w}^{ \pm}(y):=\left\langle\left(\begin{array}{c}
G_{e}(w \cdot-w d, 0 ; y) \\
G_{e}(w \cdot+w d, 0 ; y)
\end{array}\right), \boldsymbol{\Delta}_{\ell, w}^{ \pm}\left(\begin{array}{c}
u_{e}(w \cdot-w d, 0) \\
u_{e}(w \cdot+w d, 0)
\end{array}\right)\right\rangle
$$

$$
U^{w}(, k, y):=\left\langle\left(\begin{array}{c}
G_{e}(w \cdot-w d, 0 ; y) \\
G_{e}(w \cdot+w d, 0 ; y)
\end{array}\right), \mathcal{H}_{w}(k)\left(\begin{array}{c}
u_{e}(w \cdot-w d, 0) \\
u_{e}(w \cdot+w d, 0)
\end{array}\right)\right\rangle .
$$

From (5.1) and (5.2) together with the asymptotic of $\boldsymbol{\Delta}_{\ell, w}^{+}$, we obtain the expected expression for $\kappa_{\ell, w}^{+}(y)$. Using the asymptotic expansion of $H_{0}^{(1)}\left(k\left|\left(w y_{1} \pm w d, w y_{2}\right)\right|\right)$ in (5.6) we derive the asymptotic of $\kappa_{\ell, w}^{+}\left(w y_{1} \pm w d, w y_{2}\right)$.

We now treat $\kappa_{\ell, w}^{-}(y)$. By (5.1) and (5.2), one has for every $y \in \Omega_{E}$,

$$
\begin{aligned}
\left(\begin{array}{c}
G_{e}(w \cdot-w d, 0 ; y) \\
G_{e}(w \cdot+w d, 0 ; y)
\end{array}\right)= & -\frac{i}{2} H_{0}^{(1)}(k|y|)\left(e_{1}+e_{2}\right) \\
& +\left[-\eta_{1}(y)\left(x e_{1}+x e_{2}\right)+\eta_{1}(y) d\left(e_{1}-e_{2}\right)\right] w \\
& +\left[2 \eta_{2}(y) d\left(x e_{1}-x e_{2}\right)-\eta_{2}(y)\left(x^{2} e_{1}+x^{2} e_{2}\right)-\eta_{2}(y) d^{2}\left(e_{1}+e_{2}\right)\right] w^{2} \\
& +o\left(w^{2}\right) \quad \text { in }\left[H^{1 / 2}(\Gamma)\right]^{2},
\end{aligned}
$$

and

$$
\begin{aligned}
\left(\begin{array}{c}
u_{e}(w \cdot-w d, 0) \\
u_{e}(w \cdot+w d, 0)
\end{array}\right)= & -\frac{i}{2}\left(\int_{\Omega_{e}} H_{0}^{(1)}(k|z|) f(z) d z\right)\left(e_{1}+e_{2}\right) \\
& +\left[-\bar{\eta}_{1}\left(x e_{1}+x e_{2}\right)+\bar{\eta}_{1} d\left(e_{1}-e_{2}\right)\right] w \\
& +\left[2 \bar{\eta}_{2} d\left(x e_{1}-x e_{2}\right)-\bar{\eta}_{2}\left(x^{2} e_{1}+x^{2} e_{2}\right)-\bar{\eta}_{2} d^{2}\left(e_{1}+e_{2}\right)\right] w^{2} \\
& +o\left(w^{2}\right) \quad \text { in }\left[H^{1 / 2}(\Gamma)\right]^{2} .
\end{aligned}
$$

Using the symmetric structure of the problem (see Remark 4.7) we infer that

$$
\left\{\begin{array}{l}
\left\langle e_{1}+e_{2}, \mathcal{L}_{w}^{-1}\left(k_{\ell, w}^{-}\right)\left(e_{1}-e_{2}\right)\right\rangle=0, \\
\left\langle x e_{1}-x e_{2}, \mathcal{L}_{w}^{-1}\left(k_{\ell, w}^{-}\right)\left(e_{1}-e_{2}\right)\right\rangle=0, \\
\left\langle x^{2} e_{1}+x^{2} e_{2}, \mathcal{L}_{w}^{-1}\left(k_{\ell, w}^{-}\right)\left(e_{1}-e_{2}\right)\right\rangle=0,
\end{array}\right.
$$

hence by (4.26),

$$
\boldsymbol{\Delta}_{\ell, w}^{-}\left(e_{1}+e_{2}\right)=\boldsymbol{\Delta}_{\ell, w}^{-}\left(x e_{1}-x e_{2}\right)=\boldsymbol{\Delta}_{\ell, w}^{-}\left(x^{2} e_{1}+x^{2} e_{2}\right)=0 .
$$

Since

$$
\mathcal{L}_{w}^{-1}\left(k_{\ell, w}^{-}\right) \theta_{w}\left(k_{\ell, w}^{-}\right)\left(e_{1}-e_{2}\right)=\left(\frac{\alpha\left(k_{\ell, w}^{-}\right)}{w}-\delta_{1}\right) \mathcal{L}_{w}^{-1}\left(k_{\ell, w}^{-}\right)\left(e_{1}-e_{2}\right) .
$$

Using again (4.26) we obtain

$$
\begin{aligned}
\left\langle e_{1}+e_{2}, \boldsymbol{\Delta}_{\ell, w}^{-}\left(e_{1}-e_{2}\right)\right\rangle & =\left\langle x e_{1}-x e_{2}, \boldsymbol{\Delta}_{\ell, w}^{-}\left(e_{1}-e_{2}\right)\right\rangle \\
& =\left\langle x^{2} e_{1}+x^{2} e_{2}, \boldsymbol{\Delta}_{\ell, w}^{-}\left(e_{1}-e_{2}\right)\right\rangle=0,
\end{aligned}
$$

and

$$
\begin{aligned}
\left\langle e_{1}+e_{2}, \boldsymbol{\Delta}_{\ell, w}^{-}\left(x e_{1}+x e_{2}\right)\right\rangle & =\left\langle x e_{1}-x e_{2}, \Delta_{\ell, w}^{-}\left(x e_{1}+x e_{2}\right)\right\rangle \\
& =\left\langle x^{2} e_{1}+x^{2} e_{2}, \Delta_{\ell, w}^{-}\left(x e_{1}+x e_{2}\right)\right\rangle=0 .
\end{aligned}
$$


Consequently, since $\boldsymbol{\Delta}_{\ell, w}^{-}$is of order $w$ (see Theorem 4.13) we have

$$
\kappa_{\ell, w}^{-}(y)=\bar{\eta}_{1} \eta_{1}(y)\left\langle-\left(x e_{1}+x e_{2}\right)+d\left(e_{1}-e_{2}\right), \boldsymbol{\Delta}_{\ell, w}^{-}\left[-\left(x e_{1}+x e_{2}\right)+d\left(e_{1}-e_{2}\right)\right]\right\rangle w^{2}+o\left(w^{3}\right)
$$

and we obtain the desired result using the asymptotic expansion of $\boldsymbol{\Delta}_{\ell, w}^{-}$in Theorem 4.13 together with Remark 5.3. Using now the asymptotic expansion

$$
G_{e}\left(w t, 0 ; w y_{1} \pm w d, w y_{2}\right)=\frac{\ln w}{\pi}+\left[\delta+\frac{\ln k}{\pi}+\frac{\ln \left|\left(y_{1} \pm d-t, y_{2}\right)\right|}{\pi}\right]+o(w),
$$

and defining

$$
\Gamma^{-}\left(x_{1}, y_{1}, y_{2}\right):=\left(\begin{array}{l}
\ln \left|\left(y_{1}-x_{1}, y_{2}\right)\right| \\
\ln \left|\left(y_{1}-x_{1}+2 d, y_{2}\right)\right|
\end{array}\right)
$$

and

$$
\Gamma^{+}\left(x_{1}, y_{1}, y_{2}\right):=\left(\begin{array}{l}
\ln \left|\left(y_{1}-x_{1}, y_{2}\right)\right| \\
\ln \left|\left(y_{1}-x_{1}-2 d, y_{2}\right)\right|
\end{array}\right),
$$

we derive the asymptotic of $\kappa_{\ell, w}^{-}\left(w y_{1} \pm w d, w y_{2}\right)$.

Finally we argue exactly as in the proof of Theorem 5.1 to get the asymptotics in $H_{\mathrm{loc}}^{1}\left(\Omega_{e}\right)$ for the far field, and in $H^{1}\left(B_{1}^{+}\right)$for the near field.

Remark 5.5. Applying the Helmholtz operator $\Delta+k^{2}$ to $\kappa_{\ell, w}^{ \pm}$implies, according to Remark 5.3, that

$$
\left\{\begin{array}{l}
\left(\Delta+k^{2}\right) \kappa_{\ell, w}^{+}=c_{\ell}^{+} w \delta_{0}, \\
\left(\Delta+k^{2}\right) \kappa_{\ell, w}^{-}=c_{\ell}^{-} w^{3} D_{x_{1}} \delta_{0},
\end{array}\right.
$$

in $\left(C_{c}^{\infty}\left(\Omega_{e}\right)\right)^{\prime}$, where $c_{\ell}^{ \pm}$are the constants defined by

$$
c_{\ell}^{+}:=-\frac{2 u_{e}(0)}{\alpha^{\prime}\left(k_{\ell}\right)},
$$

and

$$
c_{\ell}^{-}:=-\frac{1}{2 \alpha^{\prime}\left(k_{\ell}\right)} \frac{\partial u_{e}}{\partial x_{1}}(0)\left(2 d-\frac{\left\langle x e_{2}+x e_{2}, \mathcal{S}^{-1}\left(e_{1}-e_{2}\right)\right\rangle}{q_{0}^{-}}\right)^{2} .
$$

These equations essentially say that at the frequency $k_{\ell, w}^{+}$, the spatial singularity sensed in the far field is that of a Dirac mass, which is the macroscopic manifestation of a dipole placed on a metallic plane. On the other hand, when the other resonance $k_{\ell, w}^{-}$is excited, the spatial singularity is the derivative in the $x_{1}$-direction of a Dirac mass; this is exactly the asymptotic response of a quadripole placed on the metallic plane.

Remark 5.6. The asymptotic of the field close to the resonances shows that the field $u$ concentrates on the top of the cavities as their width $w$ shrinks. Indeed,using Theorems 4.11 and 5.4, we infer that when $k$ is close to $k_{\ell, w}^{+}$, then

$$
\begin{aligned}
\frac{\kappa_{\ell, w}^{+}\left(w y_{1} \pm w d, w y_{2}\right)}{k-k_{\ell, w}^{+}}= & \frac{2 u_{e}(0)}{\pi} \ln w \\
& +2 u_{e}(0)\left(\delta+\frac{\ln k_{\ell}}{\pi}+\frac{\ln \left|\left(y_{1} \pm d, y_{2}\right)\right|}{\pi}\right)+o(1),
\end{aligned}
$$

while if $k$ is close to $k_{\ell, w}^{-}$, then

$$
\begin{aligned}
& \frac{\kappa_{\ell, w}^{-}\left(w y_{1} \pm w d, w y_{2}\right)}{k-k_{\ell, w}^{-}} \\
& \quad=\frac{4}{\pi w} \frac{\left\langle\Gamma^{ \pm}\left(\cdot, y_{1}, y_{2}\right), \mathcal{S}^{-1}\left(e_{1}-e_{2}\right)\right\rangle}{\left(2 d q_{0}^{-}-\left\langle x e_{2}+x e_{2}, \mathcal{S}^{-1}\left(e_{1}-e_{2}\right)\right\rangle\right)} \frac{\partial u_{e}}{\partial x_{1}}(0)+o(1) .
\end{aligned}
$$

In both cases, we can see that the field blows up as $w \rightarrow 0$. The concentration pattern scales like $\ln w$ close to the resonance $k_{\ell, w}^{+}$, and like $1 / w$ close to the resonance $k_{\ell, w}^{-}$. 
Remark 5.7. Thanks to the expressions of the Green functions $G_{i}$, it is possible to prove by arguments simlar to those used in the proof of Theorem 5.4 and Remarks 5.5 and 5.6, that close to the resonances, the field $u$ is asymptotically very large inside each cavity. Indeed, we can use the representation (2.8) to show (as in the proof of (5.1) ) that for every $y \in C_{1} \cup C_{2}$,

$$
u(y)=\sum_{\ell=0}^{\ell_{0}} \frac{\gamma_{\ell, w}^{+}(y)}{k-k_{\ell, w}^{+}}+\sum_{\ell=0}^{\ell_{0}} \frac{\gamma_{\ell, w}^{-}(y)}{k-k_{\ell, w}^{-}}+V^{w}(k, y),
$$

where $k \mapsto V^{w}(k, y)$ is a holomorphic function for all $y \in C_{1} \cup C_{2}$, and $V^{w}(k, \cdot) \in H^{1}\left(C_{1} \cup C_{2}\right)$ for all $k \in D_{r_{0}}^{+}$. Moreover, the functions $\gamma_{\ell, w}^{ \pm}$admit the following expansions:

$$
\gamma_{\ell, w}^{+}(y):=\frac{2 u_{e}(0)}{h \alpha^{\prime}\left(k_{\ell}\right)} \sum_{n=0}^{+\infty} \frac{(-1)^{n} \cos \left(\frac{n \pi}{h}\left(y_{2}+h\right)\right)}{k_{\ell}^{2}-\left(\frac{n \pi}{h}\right)^{2}}+O(w \ln w) \quad \text { for every } y \in C_{1} \cup C_{2},
$$

while

$$
\gamma_{\ell, w}^{-}(y):=-\frac{w}{h \alpha^{\prime}\left(k_{\ell}\right)}\left(\frac{\left\langle x e_{1}+x e_{2}, \mathcal{S}^{-1}\left(e_{1}-e_{2}\right)\right\rangle}{q_{0}^{-}}-2 d\right) \sum_{n=0}^{+\infty} \frac{(-1)^{n} \cos \left(\frac{n \pi}{h}\left(y_{2}+h\right)\right)}{k_{\ell}^{2}-\left(\frac{n \pi}{h}\right)^{2}} \frac{\partial u_{e}}{\partial x_{1}}(0)+O\left(w^{2}\right)
$$

if $y \in C_{1}$, and

$$
\gamma_{\ell, w}^{-}(y):=\frac{w}{h \alpha^{\prime}\left(k_{\ell}\right)}\left(\frac{\left\langle x e_{1}+x e_{2}, \mathcal{S}^{-1}\left(e_{1}-e_{2}\right)\right\rangle}{q_{0}^{-}}-2 d\right) \sum_{n=0}^{+\infty} \frac{(-1)^{n} \cos \left(\frac{n \pi}{h}\left(y_{2}+h\right)\right)}{k_{\ell}^{2}-\left(\frac{n \pi}{h}\right)^{2}} \frac{\partial u_{e}}{\partial x_{1}}(0)+O\left(w^{2}\right)
$$

if $y \in C_{2}$. Thus, using again Theorem 4.11, we infer that at a frequency $k$ close to $k_{\ell, w}^{+}$, one has

$$
\frac{\gamma_{\ell, w}^{+}(y)}{k-k_{\ell, w}^{+}}=\frac{a_{\ell}^{+}(y)}{w}(1+O(w \ln w)) \quad \text { for every } y \in C_{1} \cup C_{2},
$$

while at a frequency $k$ close to $k_{\ell, w}^{-}$,

$$
\frac{\gamma_{\ell, w}^{-}(y)}{k-k_{\ell, w}^{-}}=\left\{\begin{aligned}
\frac{a_{\ell}^{-}(y)}{w^{2}}(1+O(w)) & \text { if } \quad y \in C_{1}, \\
-\frac{a_{\ell}^{-}(y)}{w^{2}}(1+O(w)) & \text { if } \quad y \in C_{2},
\end{aligned}\right.
$$

where $a_{\ell}^{ \pm}(y)$ are functions defined by

$$
a_{\ell}^{+}(y):=-\frac{2 u_{e}(0)}{h} \sum_{n=0}^{+\infty} \frac{(-1)^{n} \cos \left(\frac{n \pi}{h}\left(y_{2}+h\right)\right)}{k_{\ell}^{2}-\left(\frac{n \pi}{h}\right)^{2}},
$$

and

$$
a_{\ell}^{-}(y):=\frac{4}{k_{\ell}^{2} h} \frac{\partial u_{e}}{\partial x_{1}}(0)\left(\frac{\left\langle x e_{1}+x e_{2}, \mathcal{S}^{-1}\left(e_{1}-e_{2}\right)\right\rangle}{q_{0}^{-}}-2 d\right) \sum_{n=0}^{-1} \frac{(-1)^{n} \cos \left(\frac{n \pi}{h}\left(y_{2}+h\right)\right)}{k_{\ell}^{2}-\left(\frac{n \pi}{h}\right)^{2}} .
$$

The expressions highlight the symmetric or antisymmetric nature of the modes and the field enhancement inside the cavities. Indeed, when the resonance $k_{\ell, w}^{+}$is activated, the field $u$ has the same sign in both cavities: this is the symmetrical mode. In that case, the amplification factor scales like $1 / w$, which is in agreement with [7]. On the other hand, the field $u$ has opposite signs in the cavities when the resonance $k_{\ell, w}^{-}$is excited, and the amplification factor, much larger, scales like $1 / w^{2}:$ this is the anti-symmetrical mode. Finally, we remark that the field is independent of $y_{1}$ inside the cavities, and that it increases as $y_{2}$ tends to the top of the cavities.

\section{Appendix}

Useful formulae. The following formulae can be found, e.g., in [1].

- $H_{0}^{(1)}(z)=\left\{1+\frac{2 i}{\pi}\left[\ln \left(\frac{z}{2}\right)+\gamma\right]\right\}\left(\sum_{n=0}^{+\infty} \frac{\left(-z^{2} / 4\right)^{n}}{(n !)^{2}}\right)-\frac{2 i}{\pi} \sum_{n=1}^{+\infty}\left(\sum_{m=1}^{n} \frac{1}{m}\right) \frac{\left(-z^{2} / 4\right)^{n}}{(n !)^{2}}$, where $\gamma$ is the Euler constant; 


$$
\begin{aligned}
& -\sum_{n=0}^{+\infty} \frac{1}{r^{2}-n^{2}}=\frac{1}{2 r^{2}}+\frac{\pi}{2 r} \cot (\pi r) \\
& \text { - } \sum_{n=1}^{+\infty} \frac{\cos (n \theta)}{n}=-\ln 2-\ln \left|\sin \left(\frac{\theta}{2}\right)\right| \\
& \text { - } \sum_{n=1}^{+\infty} \frac{\cos (n \theta)}{n^{2}}=\frac{\theta^{2}}{4}-\frac{\pi|\theta|}{4}+\frac{\pi^{2}}{6} .
\end{aligned}
$$

Generalized Rouché theorem. In this section, we review the main results of [12]. Let $\mathcal{G}$ and $\mathcal{H}$ be two Banach spaces and let $\mathcal{L}(\mathcal{G}, \mathcal{H})$ be the set of all bounded operators from $\mathcal{G}$ to $\mathcal{H}$. Let $U$ be an open set in $\mathbb{C}$. Suppose that $\mathcal{A}(k)$ is an operator-valued function from $U$ to $\mathcal{L}(\mathcal{G}, \mathcal{H}) ; k_{0}$ is a characteristic value of $\mathcal{A}(k)$ if

- $\mathcal{A}(k)$ is holomorphic in some neighborhood of $k_{0}$, except possibly for $k_{0}$;

- there exists a holomorphic function $\phi(k)$, from a neighborhood of $k_{0}$ to $\mathcal{G}$, such that $\phi\left(k_{0}\right) \neq 0$, $\mathcal{A}(k) \phi(k)$ is holomorphic at $k_{0}$, and $\mathcal{A}\left(k_{0}\right) \phi\left(k_{0}\right)=0$.

The function $\phi(k)$ in the above definition is called a root function of $\mathcal{A}(k)$ associated to $k_{0}$, and $\phi\left(k_{0}\right)$ is called an eigenvector. The closure of the space of eigenvectors corresponding to $k_{0}$ is denoted by $\operatorname{Ker} \mathcal{A}\left(k_{0}\right)$.

Let $\phi_{0}$ be an eigenvector corresponding to $k_{0}$. The rank of $\phi_{0}$ is the largest integer $m$ such that there exists a complex neighborhood $V\left(k_{0}\right)$ of $k_{0}$, and two holomorphic functions $\phi: V\left(k_{0}\right) \rightarrow \mathcal{G}$ and $\psi: V\left(k_{0}\right) \rightarrow \mathcal{H}$ satisfying

$$
\mathcal{A}(k) \phi(k)=\left(k-k_{0}\right)^{m} \psi(k), \quad \phi\left(k_{0}\right)=\phi_{0} \quad \text { and } \quad \psi\left(k_{0}\right) \neq 0 .
$$

Suppose that $n=\operatorname{dim} \operatorname{Ker} \mathcal{A}\left(k_{0}\right)<+\infty$ and the ranks of all vectors in $\operatorname{Ker} \mathcal{A}\left(k_{0}\right)$ are finite. A system of eigenvectors $\phi_{0}^{j}(j=1, \ldots, n)$ is called a canonical system of eigenvectors of $\mathcal{A}\left(k_{0}\right)$ if the rank of $\phi_{0}^{j}$ is the maximum of the ranks of all eigenvectors in some direct complement in $\operatorname{Ker} \mathcal{A}\left(k_{0}\right)$ of the linear space spanned by the vectors $\phi_{0}^{1}, \ldots, \phi_{0}^{j-1}$. Then we define the null multiplicity of the characteristic value of $k_{0}$ to be the sum of the ranks of $\phi_{0}^{j}(j=1, \ldots, n)$, which is denoted by $N\left(\mathcal{A}\left(k_{0}\right)\right)$.

Suppose that $\mathcal{A}^{-1}(k)$ exists and is holomorphic in some neighborhood of $k_{0}$, except possibly at this point itself. Then the number

$$
M\left(\mathcal{A}\left(k_{0}\right)\right):=N\left(\mathcal{A}\left(k_{0}\right)\right)-N\left(\mathcal{A}^{-1}\left(k_{0}\right)\right)
$$

is called the multiplicity of the characteristic value $k_{0}$.

Suppose that the Laurent expansion of $\mathcal{A}(k)$ at $k_{0}$ is given by

$$
\mathcal{A}(k)=\sum_{j \geq-s}\left(k-k_{0}\right)^{j} A_{j} .
$$

If the operators $A_{j}(j=-s, \ldots,-1)$ are finite dimensional, then $\mathcal{A}(k)$ is called finitely meromorphic at $k_{0}$. If the operator $A_{0}$ is a Fredholm one, then $\mathcal{A}(k)$ is said to be of Fredholm type at $k_{0}$.

If $\mathcal{A}(k)$ is holomorphic and invertible at $k_{0}$, then $k_{0}$ is called a regular point of $\mathcal{A}(k)$. A point $k_{0}$ is called a normal point of $\mathcal{A}(k)$ if $\mathcal{A}(k)$ is finitely meromorphic and of Fredholm type at $k_{0}$, and if there exists some neighborhood $V\left(k_{0}\right)$ of $k_{0}$ in which all the points except $k_{0}$ are regular points of $\mathcal{A}(k)$.

Lemma 6.1. Every normal point $k_{0}$ of $\mathcal{A}(k)$ is a normal point of $\mathcal{A}^{-1}(k)$.

An operator-valued function $\mathcal{A}(k)$ which is finitely meromorphic and of Fredholm type in $V\left(k_{0}\right)$ and continuous on $\partial V\left(k_{0}\right)$ is called normal with respect to $\partial V\left(k_{0}\right)$ provided it is invertible in $\overline{V\left(k_{0}\right)}$, except for a finite number of points of $V\left(k_{0}\right)$ which are normal points of $\mathcal{A}(k)$.

Suppose that $\mathcal{A}(k)$ is normal with respect to $\partial V\left(k_{0}\right)$ and let $k_{i}(i=1, \ldots, \sigma)$ be its characteristic values and poles lying in $V\left(k_{0}\right)$, we set

$$
\mathcal{M}\left(\mathcal{A}(k), \partial V\left(k_{0}\right)\right):=\sum_{i=1}^{\sigma} M(\mathcal{A}(k)) .
$$

The generalization of Rouché's Theorem is stated below: 
Theorem 6.2. Let $\mathcal{A}(k)$ be an operator-valued function which is normal with respect to $\partial V\left(k_{0}\right)$. If $\mathcal{S}(k)$ is an operator-valued function which is finitely meromorphic in $V\left(k_{0}\right)$, continuous at $\partial V\left(k_{0}\right)$ and satisfying

$$
\left\|\mathcal{A}^{-1}(k) \mathcal{S}(k)\right\|_{\mathcal{L}(\mathcal{G}, \mathcal{G})}<1 \quad \text { for } k \in \partial V\left(k_{0}\right),
$$

then $\mathcal{A}(k)+\mathcal{S}(k)$ is normal with respect to $\partial V\left(k_{0}\right)$ as well, and

$$
\mathcal{M}\left(\mathcal{A}(k), \partial V\left(k_{0}\right)\right)=\mathcal{M}\left(\mathcal{A}(k)+\mathcal{S}(k), \partial V\left(k_{0}\right)\right) .
$$

The generalization of Steinberg's Theorem is given by

Theorem 6.3. Suppose that $\mathcal{A}(k)$ is an operator-valued function which is finitely meromorphic and of Fredholm type in $V\left(k_{0}\right)$. If $\mathcal{A}(k)$ is invertible at one point of $V\left(k_{0}\right)$, then $\mathcal{A}(k)$ has a bounded inverse for all $k \in V\left(k_{0}\right)$, except possibly for certain isolated points.

We finally state a generalization of Rouché's Theorem which is also called generalized argument principle.

Theorem 6.4. Suppose that the operator-valued function $\mathcal{A}(k)$ is normal with respect to $\partial V\left(k_{0}\right)$. Let $f(k)$ be a scalar function which is holomorphic in $V\left(k_{0}\right)$ and continuous in $\overline{V\left(k_{0}\right)}$. Then we have

$$
\frac{1}{2 i \pi} \operatorname{tr} \int_{\partial V\left(k_{0}\right)} f(k) \mathcal{A}^{-1}(k) \frac{d \mathcal{A}(k)}{d k} d k=\sum_{j=1}^{\sigma} M\left(\mathcal{A}\left(k_{j}\right) f\left(k_{j}\right)\right),
$$

where $k_{j}(j=1, \ldots, \sigma)$ are all the poles or characteristic values of $\mathcal{A}(k)$ in $V\left(k_{0}\right)$.

Here tr denotes the trace of the operator which is the sum of all its nonzero eigenvalues. We mention the following property of the trace

$$
\operatorname{tr} \int_{\partial V\left(k_{0}\right)} \mathcal{A}(k) \mathcal{B}(k) d k=\operatorname{tr} \int_{\partial V\left(k_{0}\right)} \mathcal{B}(k) \mathcal{A}(k) d k,
$$

where $\mathcal{A}(k)$ and $\mathcal{B}(k)$ are operator-valued functions which are finitely meromorphic in $V\left(k_{0}\right)$, and $V\left(k_{0}\right)$ contains no pole of $\mathcal{A}(k)$ and $\mathcal{B}(k)$ other than $k_{0}$.

\section{ACKNOWLEDGEMENT}

The research of J.-F. Babadjian has been supported by the CNRS, and by the Chair "Mathematical Modelling and Numerical Simulation, F-EADS Ecole Polytechnique INRIA F-X".

\section{REFERENCES}

[1] M. Abramowitz \& I. A. Stegun: Handbook on mathematical functions with formulas, graphs and mathematical tables, National bureau of standards applied mathematics Series 55 (1972).

[2] H. Ammari, G. BAO \& A.W. Wood: Analysis of the electromagnetic scattering from a cavity, Japan J. Indust. Math. 19, no2, 2002.

[3] H. Ammari, H. Kang \& H. LeE: Layer potential techniques in spectral analysis, Mathematical Surveys and Monographs, Volume 153, American Mathematical Society, Providence, 2009.

[4] H. Ammari and F. Triki: Resonances for microstrip transmission lines, SIAM J. Appl. Math. 64, no. 2, 601-636, 2004.

[5] A. Barbara, P. Quémerais, E. Bustarret \& T. Lopez-Rios: Electromagnetic optical transmission through subwavelength metallic gratings, Phys. Rev. B 66 (2002) 161403.

[6] A. Barbara, P. Quémerais, J. Le Perchec \& T. Lopez-Rios: Efficient excitation of cavity resonances of subwavelength metallic gratings, J. appl. Phys. 97 (2005) 053507.

[7] A. Barbara, P. Quémerais, J. Le Perchec \& T. Lopez-Rios: Controlling strong electromagnetic fields at a sub-wavelength scale, Phys. Rev. Lett. 97 (2006), 036405.

[8] E. Bonnetier \& F. Triki: Asymptotic of the Green function for the diffraction by a perfectly conducting plane perturbed by a sub-wavelength rectangular cavity, Math. Meth. in the Appl. Sci. to appear (2010).

[9] M. Clausel, M. Durufle, P. Joly and S. Tordeux: A mathematical analysis of the resonance of the finite thin slots. Applied Numerical Mathematics, vol 56, no 10-11, pp 1432-1449, 2006.

[10] D. Colton And R. Kress: Inverse Acoustic and Electromagnetic Scattering Theory, Applied Mathematical Sciences 93, Springer, 1998.

[11] A. Degiron And T.W. EbBesen: The role of localized surface plsasmon modes in the enhanced transmission of periodic subwavelength apertures, J. Opt. A: Pure Appl. Opt. 7, S90-S96, 2005.

[12] I. C. Gohberg \& E. I. Sigal: An operator generalization of the logarithmic residue theorem and Rouché's theorem, Math. USSR-Sb. 13 (1971), 603-625. 
[13] P. Joly AND S. Tordeux: Matching of asymptotic expansions for wave propagation in media with thin slots I: The asymptotic expansion, SIAM M.M.S. vol. 5 (1), pp. 304-336, 2006.

14] T. Kato: Perturbation Theory for Linear Operators. Springer Verlag, New York, 1966.

[15] G. Kriegsmann: Complete Transmission Through a Two-Dimensional Diffraction Grating, SIAM J. Appl. Math., volume 65 , issue $1,24-42,2004$

[16] J. Gopalakrishnan, S. Moskow, and F. Santosa: Asymptotic and numerical techniques for resonances of thin photonic structures., SIAM J. Appl. Math., Volume 69, Issue 1, (2008) pp. 37-63.

[17] W. MCLEAN: Strongly elliptic systems and boundary integral equations, Cambridge University Press (2000).

[18] J.C. NÉDÉLEC: Electromagnetic and Acoustic Waves, Springer-Verlag, 2000.

[19] W. Rudin: Real and complex analysis, Mc Graw-Hill International Editions, 3rd edition.

[20] M. E. TAYLOR: Pseudodifferential operators, Princeton University Press (1991).

(J.-F. Babadjian) UPMC Univ PARIs 06, UMR 7598 LJLL, PARIS, F-75005 France; CNRS, UMR 7598 LJLL, PARIS, F-75005 FRANCE

E-mail address: babadjian@ann.jussieu.fr

(E. Bonnetier, F. Triki) Laboratoire Jean Kuntzmann, Université Joseph Fourier, BP 53, 38041 Grenoble Cedex 9, France

E-mail address: eric.bonnetier@imag.fr

E-mail address: faouzi.triki@imag.fr 